\title{
MÉTHODES INTRINSÈQUES POUR LE CONTRÔLE ET L'OPTIMISATION DES COQUES MINCES
}

\author{
MICHEL C. DELFOUR
}

\begin{abstract}
Cet article fait le tour d'un certain nombre de résultats récents sur une approche intrinsèque de la modélisation des coques minces en vue du contrôle et de l'optimisation de forme. Elle est basée sur le mariage de la fonction distance orientée ou algébrique et du calcul différentiel tangentiel qui sert à exprimer les notions de géométrie et de calcul différentiel sur une sous-variété de $\mathbb{R}^{N}$.
\end{abstract}

Mots-clés : géométrie intrinsèque, coques minces, contrôle, optimisation de forme.

Classification mathématique: AMS 73K15, 73K12, 49Q, 73062.

\section{INTRODUCTION}

La théorie des coques minces est un sujet classique fort bien couvert dans de nombreux ouvrages. Par exemple le lecteur peut se reporter à $[4,6,36,51,73,74]$, mais ceci ne constitue pas une liste exhaustive. La recherche dans ce domaine est présentement stimulée par un certain nombre de questions provenant d'applications au contrôle et à la conception des grandes structures spatiales, des robots flexibles, des matériaux composites, etc, où des modèles intrinsèques et mathématiquement plus faciles à manipuler seraient fort utiles.

L'objectif de cet article est de faire le tour d'un certain nombre de résultats récents sur une approche intrinsèque de la modélisation des coques minces en vue du contrôle et de l'optimisation de forme. Elle est basée sur le mariage de la fonction distance orientée ou algébrique et du calcul différentiel tangentiel qui sert à exprimer les notions de géométrie et de calcul différentiel sur une sous-variété de $\mathbb{R}^{N}$ nécessaires en théorie des coques minces. Ici les dérivées tangentielles sont introduites à partir d'un prolongement dans un voisinage de la sous-variété. Parmi tous les prolongements celui obtenu par composition avec la projection sur la sous-variété est particulièrement avantageux car la projection s'exprime explicitement en termes du gradient du carré de la fonction distance orientée. Cette approche permet de développer et de réécrire des modèles de coques minces en termes d'opérateurs différentiels intrinsèques naturels et faciles à manipuler. En fait la recherche de formulations intrinsèques indépendantes de toute paramétrisation ou bases locales n'est pas nouvelle. Elle est d'un intérêt central en géométrie et remonte au moins à Riemann. On retrouve ce point de vue fondamental et important par exemple dans les travaux de R. Valid [73, 74] et P. Germain [46].

Notre intérêt pour les coques remonte aux travaux conjoints $([25,24,30])$ avec J.P. Zolésio présentés en juin 1992 (IFIP Workshop, Sophia-Antipolis, France) sur l'utilisation de la fonction distance pour générer des topologies sur des classes d'équivalence d'ensembles, donner un sens à la continuité des 
fonctionnelles de forme et caractériser les familles compactes d'ensembles, afin d'obtenir de nouvelles formulations des problèmes et des résultats d'existence. Ces travaux ainsi que d'autres par les spécialistes des équations géométriques ([48]) révèlent que la fonction distance orientée ou algébrique permet de décrire les propriétés fines d'un ensemble et de sa frontière.

Pour illustrer ces propriétés un premier modèle linéaire rudimentaire [26] fut présenté par J.P. Zolésio à un "IMA Workshop" en novembre 1992 (Minneapolis, États-Unis) suivi d'une version plus élaborée ([27]) où le vecteur de déplacement et le tenseur linéarisé des contraintes sont approchés par des polynômes du premier ordre dans la variable normale à la surface moyenne : un modèle de type $P(1,1)$, où $P(k, \ell)$ indique une approximation polynômiale d'ordre $k \geq 0$ pour le vecteur de déplacement et d'ordre $\ell \geq 0$ pour le tenseur linéarisé des déformations.

Pour des raisons techniques nous sommes passés à un modèle de type $P(1,2)$ dans [28]. Son avantage principal est que toute la théorie statique et dynamique se fait de façon intrinsèque sans jamais revenir à des paramétrisations locales. Il préserve de façon naturelle les mouvements rigides approchés, il donne des inégalités de Korn et Poincaré globales. Tout se fait à partir des premiers principes sans référence aux modèles existants qui utilisent le calcul covariant/contravariant, les bases locales et les symboles de Christoffel.

Il devint donc désirable de pouvoir comparer ces modèles avec ceux de Naghdi et de Koiter et de trouver des correspondances explicites entre les opérateurs différentiels tangentiels intrinsèques et ceux du calcul covariant/contravariant. L'autre question qui se posait était de savoir s’il était possible d'étendre les résultats du modèles $P(1,2)$ au modèle $P(1,1)$ beaucoup plus répandu. Ces questions techniques reçurent des réponses positives dans [29] en poussant plus à fond la partie géométrie différentielle et en traduisant systématiquement en langage intrinsèque plusieurs résultats. Comme pour le modèle $P(1,2)$ on préserve les mouvements rigides approchés.

Il restait un point important à vérifier pour valider ces modèles. Y avait-il convergence vers les modèles asymptotiques reconnus et plus particulièrement le modèle de coque membranaire avec les bons coefficients? Ici encore la réponse est positive. Sous des hypothèses très faibles sur le membre de droite du modèle $P(1,1)$, on obtient dans [33] la convergence des solutions (dans les espaces quotient associés aux opérateurs et aux conditions aux limites) vers celles du modèle asymptotique $P(1,0)$ qui donne à la fois l'équation de la coque membranaire avec les bons coefficients et la condition de LoveKirchhoff. Les modèles non-linéaires du type $P(1,2)$ ont aussi été abordés dans ce cadre dans [75] et [23].

Un premier effort de synthèse pour regrouper tous ces résultats dans un même cadre a été fait dans [34] sous forme de notes de cours. Nous espérons que malgré leurs lacunes elles permettront de gagner des adeptes et de servir de point de départ à des applications plus ambitieuses. C'est un peu pour cela que nous ne nous sommes pas limités à la dimension 3 , mais que nous avons tout fait en dimension $N$. Se limiter à la dimension $N=3$ ne simplifie en rien la théorie des coques minces.

Enfin, pour des raisons d'espace nous renvoyons aux articles ci-haut mentionnés pour la discussion des travaux existants dans la littérature scientifique et celle de l'ingénieur ainsi que leur comparaison avec l'ensemble des 
résultats annoncés dans cet article. Nous avons fait un effort pour regrouper la majorité des articles pertinents dans la liste de références, mais, étant néophytes dans le domaine des plaques et des coques, il y a probablement des omissions involontaires. Nous nous en excusons.

Notations. Le produit scalaire dans $\mathbb{R}^{N}$ sera

$$
x \cdot y=\sum_{i=1}^{N} x_{i} y_{i}, \quad|x|=\sqrt{x \cdot x} .
$$

On écrira ${ }^{*} v$ et ${ }^{*} A$ les transposés d'un vecteur $v$ et d'une matrice $A ; A^{-1}$ et ${ }^{*} A^{-1}$ seront les inverses de $A$ et de ${ }^{*} A$. On associe aussi à une transformation linéaire $A: \mathbb{R}^{N} \rightarrow \mathbb{R}^{K}$ la norme

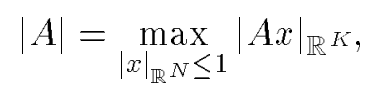

et si $A_{i j}$ and $B_{i j}$ sont les éléments de la représentation matricielle de $A$ et $B$ par rapport à des bases données $\left\{a_{1}, \ldots, a_{N}\right\}$ et $\left\{b_{1}, \ldots, b_{K}\right\}$ le produit scalaire double est défini comme

$$
A \cdot B=\sum_{i=1}^{N} \sum_{j=1}^{K} A_{i j} B_{i j} .
$$

La norme $|A|$ est équivalente à la norme $\sqrt{A \cdot A}$.

\section{Quelques notions DE GÉomÉtrie difFÉREntielle}

Une coque mince est caractérisée par un domaine borné ouvert $\omega$ dans une sous-variété $\Gamma$ qui est (au moins localement) la frontière d'un domaine $\Omega$ de classe $C^{1,1}$ dans $\mathbb{R}^{N}$ (en pratique $N=3$ ). On associe à $\Omega$ la fonction distance $d_{\Omega}$ et la fonction distance orientée $b_{\Omega}$

$$
d_{\Omega}(x) \stackrel{\text { def }}{=} \inf _{y \in \Omega}|y-x|, \quad b_{\Omega}(x) \stackrel{\text { def }}{=} d_{\Omega}(x)-d_{\mathbb{R}^{N-\Omega}}(x), \quad \forall x \in \mathbb{R}^{N} .
$$

On suppose désormais $\Gamma \stackrel{\text { def }}{=} \partial \Omega$. On a la propriété remarquable suivante : pour $k \geq 1$

$$
\Omega \text { est de classe } C^{k, 1} \Longleftrightarrow \begin{gathered}
\exists \text { un voisinage } N(\Gamma) \text { de } \Gamma \\
\text { tel que } b_{\Omega} \in C^{k, 1}(N(\Gamma)) .
\end{gathered}
$$

Le gradient $\nabla b_{\Omega}$ coïncide avec la normale extérieure unitaire $n$ sur $\Gamma$. La projection $p_{\Gamma}$ sur $\Gamma$ et la projection orthonormale $P_{\Gamma}$ sur le plan tangent $T_{x} \Gamma$ sont données par

$$
p_{\Gamma}(x)=x-b_{\Omega}(x) \nabla b_{\Omega}(x), \quad P_{\Gamma}(x)=I-\nabla b_{\Omega}(x)^{*} \nabla b_{\Omega}(x) .
$$

Pour $b_{\Omega} \in C^{1,1}(N(\Gamma))$, les éléments de $D^{2} b_{\Omega}$ appartiennent à $W^{1, \infty}(N(\Gamma))$ et les valeurs propres de $D^{2} b_{\Omega}$ sont les $(N-1)$ courbures principales $\left\{\kappa_{\alpha}\right\}$ de $\Gamma$ plus 0 . De là, la courbure moyenne (au facteur multiplicatif $(N-1)$ près) et la courbure de Gauss sont données par

$$
H=\sum_{\alpha=1}^{N-1} \kappa_{\alpha}=\Delta b_{\Omega} \quad \text { et } \quad K=\prod_{\alpha=1}^{N-1} \kappa_{\alpha} .
$$

Puisque $\bar{\omega}$ est compact il existe $h>0$ tel que $b_{\Omega} \in C^{1,1}\left(\overline{S_{h}(\omega)}\right)$ où

$$
S_{h}(\omega) \stackrel{\text { def }}{=}\left\{x \in \mathbb{R}^{N}:\left|b_{\Omega}(x)\right|<h, p_{\Gamma}(x) \in \omega\right\} .
$$


Lorsque $\omega=\Gamma$, la coque n'a pas de frontière ; sinon, on désigne par $\gamma$ la frontière de $\omega$ dans $\Gamma$ et par

$$
\Sigma_{h}(\gamma) \stackrel{\text { def }}{=}\left\{x \in \mathbb{R}^{N}:\left|b_{\Omega}(x)\right|<h, p_{\Gamma}(x) \in \gamma\right\}
$$

la frontière latérale de $S_{h}(\omega)$. On associe aussi à $\gamma_{0} \subset \gamma$

$$
\Sigma_{h}\left(\gamma_{0}\right) \stackrel{\text { def }}{=}\left\{x \in \mathbb{R}^{N}:\left|b_{\Omega}(x)\right|<h, p_{\Gamma}(x) \in \gamma_{0}\right\} .
$$

La régularité de $\gamma$ est spécifiée par la régularité de $\Sigma_{h}(\gamma)$ dans un voisinage de $\gamma$. Par exemple, $\gamma$ est lipschitzienne ou $C^{k}$ (resp. possède la condition de cône uniforme) si $\Sigma_{h}(\gamma)$ est lipschitzienne ou $C^{k}$ (resp. possède la condition de cône uniforme) dans un voisinage de $\gamma$. Une autre propriété importante lorsque $b_{\Omega} \in C^{1,1}\left(\overline{S_{h}(\omega)}\right)$ est que l'application

$$
\left.(X, z) \mapsto T(X, z) \stackrel{\text { def }}{=} X+z \nabla b_{\Omega}(X): \omega \times\right]-h, h\left[\rightarrow S_{h}(\omega)\right.
$$

et son inverse $T^{-1}(x)=\left(p_{\Gamma}(x), b_{\Omega}(x)\right)$ sont toutes deux lipschitziennes. On utilisera aussi la notation

$$
T_{z}(X) \stackrel{\text { def }}{=} T(X, z)=X+z \nabla b_{\Omega}(X) .
$$

Ceci induit un isomorphisme linéaire et continu

$$
f \mapsto f \circ T: L^{2}(\omega \times]-h, h[) \rightarrow L^{2}\left(S_{h}(\omega)\right) .
$$

Le polynôme suivant interviendra dans le changement de variables le long des ensembles de niveau de $b_{\Omega}$ : pour tout $|z|<h, X \in \omega$, on définit

$$
j_{z}(X) \stackrel{\text { def }}{=} \operatorname{det} D T_{z}(X)=\operatorname{det}\left[I+z D^{2} b_{\Omega}(X)\right]=\sum_{i=0}^{N-1} \lambda_{i}(X) z^{i},
$$

où les fonctions $\lambda_{i}(X)$ sont les coefficients du polynôme en $z$.

Puisque maintenant $\Omega$ et $\Gamma$ seront fixés, nous omettons les indices de $b$, $p$ et $P$. Il y a plusieurs façons d'introduire les dérivées dans $\Gamma$. Par exemple les dérivées covariantes/contravariantes par rapport à un système de bases locales associé à une carte ou encore l'utilisation d'une connexion riemanienne. Afin d'éviter le phénomène de paramétrisation, il est plus naturel de choisir les dérivées tangentielles qui sont l'objet intrinsèque sousjacent à toutes les notions de dérivées dans $\Gamma$. Le gradient tangentiel d'une fonction $f: \omega \rightarrow \mathbb{R}$ est défini via un prolongement $F: S_{h}(\omega) \rightarrow \mathbb{R}$ de $f$ par

$$
\left.\nabla_{\Gamma} f \stackrel{\text { def }}{=} \nabla F\right|_{\Gamma}-\frac{\partial F}{\partial n} n .
$$

Ce vecteur est indépendant $\mathrm{d} u$ choix $\mathrm{d} u$ prolongement. Parmi tous ces prolongements $F=f \circ p$ est particulièrement intéressant puisque

$$
\nabla_{\Gamma} f=\left.\nabla(f \circ p)\right|_{\omega} .
$$

À l'aide de cette propriété le calcul différentiel dans la sous-variété $\Gamma$ s'obtient du calcul différentiel habituel dans le voisinage $S_{h}(\omega)$ par restriction à $\Gamma$. Pour le cas $C^{1,1}$ on peut alors définir l'espace de Sobolev $H^{1}$ intrinsèquement. De plus, l'isomorphisme (6) s'étend aux espaces $H^{1}$

$$
f \mapsto f \circ T: H^{1}(\omega \times]-h, h[) \rightarrow H^{1}\left(S_{h}(\omega)\right) .
$$

En particulier, $H^{1}(\omega \times]-h, h[)=H^{1}\left(-h, h ; L^{2}(\omega)\right) \cap L^{2}\left(-h, h ; H^{1}(\omega)\right)$. Il y a d'autres propriétés utiles comme

$$
\nabla(f \circ p)=\left[I-b D^{2} b\right] \nabla_{\Gamma} f \circ p .
$$


La matrice jacobienne tangentielle d'une fonction $v: \omega \rightarrow \mathbb{R}$ est donnée par

$$
\left.D_{\Gamma}(v) \stackrel{\text { def }}{=} D(v \circ p)\right|_{\Gamma} \text { ou }\left(D_{\Gamma} v\right)_{i j}=\left(\nabla_{\Gamma} v_{i}\right)_{j}
$$

Si ${ }^{*} v=\left(v_{1}, \ldots, v_{M}\right)$, alors ${ }^{*} D_{\Gamma} v=\left(\nabla_{\Gamma} v_{1}, \ldots, \nabla_{\Gamma} v_{M}\right)$, où $\nabla_{\Gamma} v_{i}$ est un vecteur colonne. On retrouve aussi

$$
D(v \circ p)=D_{\Gamma}(v) \circ p\left[I-b D^{2} b\right], \quad D(v \circ p) \nabla b=0 \text { et } D_{\Gamma}(v) n=0 .
$$

De la même façon, on a la divergence tangentielle

$$
\left.\operatorname{div}_{\Gamma} v \stackrel{\text { def }}{=} \operatorname{div}(v \circ p)\right|_{\Gamma}
$$

et le tenseur linéarisé tangentiel des déformations

$$
\varepsilon_{\Gamma}(v) \stackrel{\text { def }}{=} \frac{1}{2}\left(D_{\Gamma} v+{ }^{*} D_{\Gamma} v\right) \quad \text { et } \quad \varepsilon_{\Gamma}(v)=\left.\varepsilon(v \circ p)\right|_{\Gamma} .
$$

La matrice jacobienne tangentielle de la normale $n$ est particulière, puisque $n \circ p=n=\nabla b=\nabla b \circ p$ et $b \circ p=0$. D'où

$$
D_{\Gamma}(n)=D_{\Gamma}(\nabla b)=\left.D^{2} b\right|_{\Gamma}={ }^{*} D_{\Gamma}(\nabla b)={ }^{*} D_{\Gamma}(n)
$$

et puisqu'elle est symétrique on a aussi

$$
\varepsilon_{\Gamma}(n)=\varepsilon_{\Gamma}(\nabla b)=\left.D^{2} b\right|_{\Gamma} .
$$

L'opérateur de Laplace-Beltrami d'une function $f$ devient

$$
\Delta_{\Gamma} f \stackrel{\text { def }}{=} \operatorname{div}_{\Gamma}\left(\nabla_{\Gamma} f\right)=\left.\Delta(f \circ p)\right|_{\Gamma} .
$$

Il est naturel d'introduire la matrice jacobienne tangentielle projetée et le tenseur linéarisé tangentiel des déformations projeté

$$
D_{\Gamma}^{P}(v) \stackrel{\text { def }}{=} P D_{\Gamma}(v) P, \quad \varepsilon_{\Gamma}^{P}(v) \stackrel{\text { def }}{=} P \varepsilon_{\Gamma}(v) P .
$$

Il est facile de vérifier que

$$
\begin{gathered}
D_{\Gamma}^{P}(v)=P D_{\Gamma}(v), \quad \varepsilon_{\Gamma}^{P}(v)=\frac{1}{2}\left(D_{\Gamma}^{P}(v)+{ }^{*} D_{\Gamma}^{P}(v)\right), \\
D_{\Gamma}(v)=D_{\Gamma}^{P}(v)+n^{*}\left({ }^{*} D_{\Gamma}(v) n\right) .
\end{gathered}
$$

Il sera souvent utile de parler de la composante normale et de la partie tangente d'un vecteur $v$ et d'utiliser la notation

$$
v_{n} \stackrel{\text { def }}{=} v \cdot n, \quad v_{\Gamma} \stackrel{\text { def }}{=} v-v_{n} n=P v
$$

On obtient alors les identités

$$
D_{\Gamma}^{P}(v)=D_{\Gamma}^{P}\left(v_{\Gamma}\right)+v_{n} D^{2} b \quad \text { et } \quad \varepsilon_{\Gamma}^{P}(v)=\varepsilon_{\Gamma}^{P}\left(v_{\Gamma}\right)+v_{n} D^{2} b .
$$

Les dérivées covariantes/contravariantes ne sont que l'expression par rapport aux bases locales des opérateurs différentiels tangentiels correspondants. Pour plus de détails le lecteur peut se reporter à [34]. 


\section{DÉVELOPPEMENT PAR RAPPORT À LA VARIABLE D'ÉPAISSEUR}

On utilisera trois jeux d'hypothèses. Soit $\omega$ un domaine borné ouvert dans $\Gamma$. Si $\omega=\Gamma$, $\omega$ est sans frontière ; sinon on suppose que $\gamma$ est lipschitzienne. Dans les deux cas on utilisera l'espace $H^{1}\left(S_{h}(\omega)\right)^{N}$. Le second cas correspond à des conditions homogènes de Neumann. Pour les conditions homogènes de Dirichlet sur tout $\Sigma_{h}(\gamma)$, on utilisera $H_{\gamma}^{1}\left(S_{h}(\omega)\right)^{N}$ avec

$$
H_{\gamma}^{1}\left(S_{h}(\omega)\right)=\left\{f \in H^{1}\left(S_{h}(\omega)\right): f=0 \operatorname{sur} \Sigma_{h}(\gamma)\right\} .
$$

Enfin, pour des conditions homogènes de Dirichlet sur une partie $\gamma_{0} \mathrm{du}$ bord $\gamma$ de $(N-1)$-capacité non nulle, on supposera $\omega$ connexe et on prendra $H_{\gamma_{0}}^{1}\left(S_{h}(\omega)\right)^{N}$ avec

$$
H_{\gamma_{0}}^{1}\left(S_{h}(\omega)\right)=\left\{f \in H^{1}\left(S_{h}(\omega)\right): f=0 \text { sur } \Sigma_{h}\left(\gamma_{0}\right)\right\} .
$$

Hypothèse 3.1. On se fixe une transformation linéaire bijective $C$ qui transforme les matrices ou tenseurs symétriques $N \times N$ en matrices ou tenseurs symétriques $N \times N$. On suppose également l'existence d'un $\nu>0$ tel que $C^{-1} \tau \cdots \tau \geq \nu\|\tau\|^{2}$ pour tous $\tau$.

Par exemple, si $\mu>0$ and $\lambda \geq 0$ sont les constantes de Lamé

$$
C^{-1} \tau=2 \mu \tau+\lambda \operatorname{tr} \tau I \text { et } \nu=2 \mu \text {. }
$$

Soit le problème $N$-dimensionnel caractérisé par l'équation variationnelle

$$
\begin{gathered}
\exists V(h) \in V\left(S_{h}(\omega)\right)^{N} \text { tel que } \forall \bar{V} \in V\left(S_{h}(\omega)\right)^{N} \\
\int_{S_{h}(\omega)} C^{-1} \varepsilon(V(h)) \cdots \varepsilon(\bar{V})-F \cdot \bar{V} d x=0
\end{gathered}
$$

pour $\varepsilon(V)=1 / 2\left(D V+{ }^{*} D V\right)$ et $V\left(S_{h}(\omega)\right)^{N}$ égal à $H_{\gamma_{0}}^{1}\left(S_{h}(\omega)\right)^{N}, H_{\gamma}^{1}\left(S_{h}(\omega)\right)^{N}$ ou $H^{1}\left(S_{h}(\omega)\right)^{N} / \operatorname{ker} \varepsilon$. Dans ce dernier cas, il faut ajouter la condition

$$
\forall \bar{V} \in \operatorname{ker} \varepsilon, \quad \int_{S_{h}(\omega)} F \cdot \bar{V} d x=0
$$

pour que $F$ définisse une forme linéaire continue dans $H^{1}\left(S_{h}(\omega)\right)^{N} /$ ker $\varepsilon$. Dans tous les cas de figure, il existe une constante $c=c\left(S_{h}(\omega)\right)>0$ telle que

$$
\forall V \in V\left(S_{h}(\omega)\right)^{N}, \quad\left|\int_{S_{h}(\omega)} F \cdot V d x\right| \leq c\|\varepsilon(V)\|_{L^{2}\left(S_{h}(\omega)\right)} .
$$

On utilise l'inégalité de Poincaré dans les cas de Dirichlet et le fait que la semi-norme $\|\varepsilon(V)\|$ devient une norme équivalente à la norme quotient dans l'autre cas.

Comme $h>0$ va tendre vers zéro, on exprime le vecteur de déplacement $V$ par rapport aux coordonnées locales à l'aide du changement de variables $T$. En supposant que $v=V \circ T$ est assez régulier

$$
\begin{gathered}
v(X, z)=\sum_{i=0}^{\infty} v^{i}(X) z^{i}, \quad v^{i}: \omega \rightarrow \mathbb{R}^{N} \\
V(x)=\left(v \circ T^{-1}\right)(x)=\sum_{i=0}^{\infty}\left(v^{i} \circ p\right)(x) b(x)^{i} .
\end{gathered}
$$

On résume les hypothèses et on choisit $h$ assez petit pour vérifier l'hypothèse suivante. 
Hypothìse 3.2 . Soit $\Gamma$ la frontière d'un domaine $\Omega$ de $\mathbb{R}^{N}$ et soit $\omega$ un domaine ouvert borné dans $\Gamma$. Soit $h>0$ tel que $b_{\Omega} \in C^{1,1}\left(\overline{S_{h}(\omega)}\right)$ et que

$$
\exists \beta, 0<\beta<1, \forall x \in \omega, \quad h\left\|D^{2} b_{\Omega}(X)\right\| \leq \beta .
$$

La matrice $\left[I+z D^{2} b\right]^{-1}$ peut alors s'écrire comme une somme

$$
\left[I+z D^{2} b\right]^{-1}=\sum_{i=0}^{\infty}\left(-D^{2} b\right)^{i} z^{i}
$$

Après substitution

$$
D V \circ T_{z}=\sum_{i=0}^{\infty} z^{i}\left\{(i+1)\left(v^{i+1 *} n\right)+\sum_{k=0}^{i} D_{\Gamma}\left(v^{k}\right)\left(-D^{2} b\right)^{i-k}\right\}
$$

et le tenseur linéarisé des déformations devient

$$
\begin{gathered}
\varepsilon(V) \circ T_{z}=\sum_{i=1}^{\infty} \varepsilon^{i}(v) z^{i} \\
2 \varepsilon^{i}(v) \stackrel{\text { def }}{=}(i+1)\left[v^{i+1 *} n+n^{*} v^{i+1}\right] \\
+\sum_{k=0}^{i}\left[D_{\Gamma}\left(v^{k}\right)\left(-D^{2} b\right)^{i-k}+\left(-D^{2} b\right)^{i-k *} D_{\Gamma}\left(v^{k}\right)\right]
\end{gathered}
$$

On utilise ensuite la décomposition de Federer de la mesure le long des ensembles de niveau de la fonction $b$ pour exprimer l'énergie de déformation comme une intégrale sur $\omega$. La forme bilinéaire dans (18) donne

$$
\int_{S_{h}(\omega)} C^{-1} \varepsilon \cdot \bar{\varepsilon} d x=\sum_{i=0}^{\infty} \int_{\omega} \alpha_{i}(h) \sum_{j=0}^{i} C^{-1} \varepsilon^{j} \cdot \cdot \bar{\varepsilon}^{i-j} d \Gamma
$$

où $\varepsilon=\varepsilon\left(\left\{v_{i}\right\}\right), \bar{\varepsilon}=\varepsilon\left(\left\{\bar{v}_{i}\right\}\right)$ et pour $n \geq 0$

$$
\alpha_{n}(h)=\int_{-h}^{h} j_{z} z^{n} d z=h^{n+1} \sum_{i=0}^{N-1}\left[1-(-1)^{n+i+1}\right] \frac{h^{i}}{n+i+1} \lambda_{i} .
$$

Ce sont des polynômes de puissances impaires de $h$. On suppose aussi que la force $F$ est de la forme

$$
F=f \circ T^{-1}, \quad f=\sum_{i=0}^{\infty} f_{i} z^{i}, \quad f_{i} \in L^{2}(\omega)^{N}, \quad \sum_{i=0}^{\infty} h^{2 i}\left\|f_{i}\right\|_{L^{2}(\omega)}^{2}<\infty .
$$

D'où

$$
\int_{S_{h}(\omega)} F \cdot V d x=\sum_{i=0}^{\infty} \int_{\omega}\left\{\alpha_{i}(h) \sum_{j=0}^{i} f^{i-j} \cdot v^{j} d z\right\} d \Gamma .
$$

Finalement il vient de (18)

$$
\begin{aligned}
& 0=\int_{S_{h}(\omega)} C^{-1} \varepsilon(V) \cdot \varepsilon(\bar{V})-F \cdot V d x \\
& 0=\sum_{i=0}^{\infty} \int_{\omega} \alpha_{i}(h) \sum_{j=0}^{i}\left\{C^{-1} \varepsilon^{i-j}(v) \cdot \varepsilon^{j}(\bar{v})-f^{i-j} \cdot \bar{v}^{j}\right\} d \Gamma .
\end{aligned}
$$




\section{LES MODÈLES POLYNÔMIAUX}

Les identités (27) sont vérifiées pour tous $\bar{V}$ et $\bar{v}$. Il est d'usage de procéder par approximation polynômiale du vecteur de déplacement $v$ et du tenseur linéarisé des déformations $\varepsilon \circ T_{z}$ par des polynômes en $z$ de degrés respectifs $k \geq 0$ et $\ell \geq 0$. On appellera $P(k, \ell)$ ce type de modèle. Les modèles $P(1,2)$ et $P(1,1)$ correspondent à une approximation de $V$ et $\bar{V}$ dans $(27)$ par

$$
V^{0}=v^{0} \circ p+b v^{1} \circ p, \quad \bar{V}^{0}=\bar{v}^{0} \circ p+b \bar{v}^{1} \circ p
$$

pour $\left(\bar{v}^{0}, \bar{v}^{1}\right)$ et $\left(v^{0}, v^{1}\right)$ définis dans $\omega$ et de $\varepsilon\left(V^{0}\right) \circ T_{z}$ par

$$
\begin{gathered}
\varepsilon\left(V^{0}\right) \circ T_{z} \simeq \varepsilon^{0}\left(v^{0}, v^{1}\right)+z \varepsilon^{1}\left(v^{0}, v^{1}\right)+z^{2} \varepsilon^{2}\left(v^{0}, v^{1}\right) \text { pour } P(1,2), \\
\varepsilon\left(V^{0}\right) \circ T_{z} \simeq \varepsilon^{0}\left(v^{0}, v^{1}\right)+z \varepsilon^{1}\left(v^{0}, v^{1}\right) \text { pour } P(1,1) .
\end{gathered}
$$

Si $v^{i}=0, i \geq 2$, les expressions (23) de $\varepsilon^{i}$ se simplifient

$$
\begin{gathered}
\varepsilon^{0}\left(v^{0}, v^{1}\right)=\frac{1}{2}\left(v^{1 *} n+n^{*} v^{1}\right)+\varepsilon_{\Gamma}\left(v^{0}\right), \text { et pour } i \geq 1 \\
\varepsilon^{i}\left(v^{0}, v^{1}\right)=\frac{1}{2} \sum_{k=0}^{1}\left[D_{\Gamma}\left(v^{k}\right)\left(-D^{2} b\right)^{i-k}+\left(-D^{2} b\right)^{i-k *} D_{\Gamma}\left(v^{k}\right)\right]
\end{gathered}
$$

\section{LE MODÈLE LINÉAIRE $P(1,1)$}

Pour alléger l'exposé on se limite à une coque $S_{h}(\omega)$ d'épaisseur constante, mais presque tout ce qui suit se généralise à des coques d'épaisseur variable. Les résultats qui suivent ont d'abord été obtenus pour le modèle $P(1,2)$. Leur prolongement au modèle $P(1,1)$ est nouveau et on trouvera le détail des démonstrations dans [29] et [34] (pour le modèle $P(1,2)$ voir [28]).

Hypothèse 5.1. En chaque point $x$ de la coque le vecteur de déplacement $V(x)$ est de la forme

$$
V(x)=e \circ p(x)+b(x) \ell \circ p(x), \quad x \in S_{h}(\omega),
$$

oì la notation a été changée de $\left(v^{0}, v^{1}\right)$ à $(e, \ell)$.

Le tenseur linéarisé des déformations est donné par

$$
\varepsilon(V) \circ T_{z}=\sum_{i=0}^{\infty} \varepsilon^{i}(e, \ell) z^{i}
$$

avec $\varepsilon^{i}(e, \ell)$ défini par (28).

\subsection{Mouvements Rigides apProchés}

Le résultat suivant est central au développement $\mathrm{d} u$ modèle.

Théorème 1 (Mouvements rigides approchés). Sous l'Hypothèse 3.2 pour e et $\ell$ dans $H^{1}(\omega)^{N}$ et $V=e \circ p+b \ell \circ p$.,

(i)

$$
\varepsilon(V)=0 \text { dans } S_{h}(\omega)
$$

si et seulement si

$$
\varepsilon^{0}(e, \ell)=\varepsilon^{1}(e, \ell)=\varepsilon^{2}(e, \ell)=0 \text { dans } \omega,
$$

ou encore si $\omega$ est connexe, si et seulement si il existe un vecteur a $\in$ $\mathbb{R}^{N}$ et une $N \times N$ matrice $B$ tel que

$$
e(X)=a+B X, \quad \ell(X)=B \nabla b(X), \quad X \in \omega,
$$


avec $B$ antisymétrique

$$
B+{ }^{*} B=0
$$

En particulier (33) et (34) impliquent que $\ell$ est tangentiel.

(ii) Pour tous $z,|z|<h, X \in \omega$,

$$
\begin{aligned}
& \varepsilon(V) \circ T \\
& \quad=\varepsilon^{0}+\left[I+z D^{2} b\right]^{-1}\left\{z\left[\varepsilon^{1}+\varepsilon^{1} z D^{2} b+z D^{2} b \varepsilon^{1}\right]+z^{2} \varepsilon^{2}\right\}\left[I+z D^{2} b\right]^{-1} .
\end{aligned}
$$

(iii) $\operatorname{ker} \varepsilon^{0} \cap \operatorname{ker} \varepsilon^{1}=\operatorname{ker} \varepsilon^{0} \cap \operatorname{ker} \varepsilon^{1} \cap \operatorname{ker} \varepsilon^{2}$.

Corollaire 2. Sous les hypothèses du théorème

$$
\begin{aligned}
& \operatorname{ker} \varepsilon^{0} \cap \operatorname{ker} \varepsilon^{1}=\operatorname{ker} \varepsilon^{0} \cap \operatorname{ker} \varepsilon^{1} \cap \operatorname{ker} \varepsilon^{2} \\
& \quad=\left\{\left(e,-{ }^{*} D_{\Gamma}(e) n\right): e \in H^{1}(\omega)^{N}, \varepsilon_{\Gamma}^{P}(e)=0, \sum_{k=1}^{N} n_{k} P D_{\Gamma}^{2}\left(e_{k}\right)=0\right\} .
\end{aligned}
$$

\subsection{Les espaces $\mathcal{H}, \mathcal{V}, \mathcal{N}$}

En vertu du Théorème 1 il est naturel d'introduire les espaces suivants

$$
\begin{aligned}
\mathcal{H} & =L^{2}(\omega)^{N} \times L^{2}(\omega)^{N}, \\
\mathcal{V} & =\left\{(e, \ell) \in \mathcal{H}: \varepsilon^{i}(e, \ell) \in L^{2}(\omega)^{N \times N}, 0 \leq i \leq 2\right\}, \\
\mathcal{N} & =\left\{(e, \ell): \varepsilon^{i}(e, \ell)=0 \text { sur } \omega, 0 \leq i \leq 2\right\},
\end{aligned}
$$

et les normes

$$
\begin{aligned}
|(e, \ell)|_{\mathcal{H}}^{2} & =|e|_{L^{2}(\omega)}^{2}+|\ell|_{L^{2}(\omega)}^{2} \\
\|(e, \ell)\|_{\mathcal{V}}^{2} & =|(e, \ell)|_{\mathcal{H}}^{2}+\sum_{i=0}^{2}\left\|\varepsilon^{i}(e, \ell)\right\|_{L^{2}(\omega)}^{2}
\end{aligned}
$$

D’après le Théorème 1 (i)

$$
\begin{aligned}
\mathcal{N}=\{(e, \ell): \ell(X)= & B \nabla b(X), e(X)=a+B X, \forall a \in \mathbb{R}^{N}, \\
& \left.\forall B \text { une } N \times N \text { matrice telle que } B+{ }^{*} B=0\right\} .
\end{aligned}
$$

Le sous-espace $\mathcal{N}$ caractérise les mouvements rigides approchés comme en élasticité 3-D ce qui est une propriété mécanique fondamentale. Noter que la semi-norme pour l'espace $\mathcal{V}$

$$
\|\mid(e, \ell)\| \|=\left\{\sum_{i=0}^{2}\left\|\varepsilon^{i}(e, \ell)\right\|_{L^{2}(\omega)}^{2}\right\}^{1 / 2},
$$

devient une norme pour l'espace quotient $\mathcal{H} / \mathcal{N}$.

ThÉORÈme 3. Soit $\omega$ un domaine borné ouvert dans $\Gamma$ qui est la frontière d'un domaine $\Omega$ de classe $C^{1,1}$ de $\mathbb{R}^{N}$ dans un voisinage de $\omega$.

(i) L'espace

$$
\left\{(e, \ell) \in \mathcal{H}: \varepsilon^{0}(e, \ell) \in L^{2}(\omega)^{N \times N}\right\},
$$

avec norme

$$
\|e\|_{L^{2}}+\|\ell\|_{L^{2}}+\left\|\varepsilon^{0}(e, \ell)\right\|_{L^{2}}
$$

est égal à $H^{1}(\omega)^{N} \times L^{2}(\omega)^{N}$ avec norme équivalente $\|\ell\|_{H^{1}}+\|\ell\|_{L^{2}}$.

(ii) Les espaces suivants sont égaux avec normes équivalentes :

a) $H^{1}(\omega)^{N} \times H^{1}(\omega)^{N}$ avec norme $\|e\|_{H^{1}}+\|\ell\|_{H^{1}}$. 
b) $\left\{(e, \ell) \in \mathcal{H}: \varepsilon^{i}(e, \ell) \in L^{2}(\omega)^{N \times N}, i=0,1\right\}$ avec norme

$$
\|e\|_{L^{2}}+\|\ell\|_{L^{2}}+\left\|\varepsilon^{0}(e, \ell)\right\|_{L^{2}}+\left\|\varepsilon^{1}(e, \ell)\right\|_{L^{2}} .
$$

c) $\mathcal{V}=\left\{(e, \ell) \in \mathcal{H}: \varepsilon^{i}(e, \ell) \in L^{2}(\omega)^{N \times N}, i=0,1,2\right\}$ avec norme

$$
\|e\|_{L^{2}}+\|\ell\|_{L^{2}}+\left\|\varepsilon^{0}(e, \ell)\right\|_{L^{2}}+\left\|\varepsilon^{1}(e, \ell)\right\|_{L^{2}}+\left\|\varepsilon^{2}(e, \ell)\right\|_{L^{2}} \text {. }
$$

d) $\left\{(e, \ell) \in \mathcal{H}: \varepsilon^{0}(e, \ell) \in L^{2}(\omega)^{N \times N}\right.$ et $\left.\varepsilon_{\Gamma}(\ell) \in L^{2}(\omega)^{N \times N}\right\}$ avec norme

$$
\|e\|_{L^{2}}+\|\ell\|_{L^{2}}+\left\|\varepsilon^{0}(e, \ell)\right\|_{L^{2}}+\left\|\varepsilon_{\Gamma}(\ell)\right\|_{L^{2}} .
$$

(iii) La semi-norme

$$
\left\|\varepsilon^{0}(e, \ell)\right\|_{L^{2}}+\left\|\varepsilon^{1}(e, \ell)\right\|_{L^{2}}
$$

est équivalente à la semi-norme

$$
\left\|\varepsilon^{0}\left(e, \ell_{\Gamma}\right)\right\|_{L^{2}}+\left\|\varepsilon^{1}\left(e, \ell_{\Gamma}\right)\right\|_{L^{2}}+\left\|\ell_{n}\right\|_{H^{1}} .
$$

\subsection{Espaces $\mathcal{V}_{0}, \mathcal{V}_{\gamma_{0}}$, et inégalité de Poincaré globale}

Dans ce paragraphe $\omega$ est un domaine ouvert borné dans $\Gamma$ de frontière lipschitzienne $\gamma$. Pour l'étude des conditions homogènes de Dirichlet sur toute la frontière, on introduit les espaces suivants

$$
\begin{aligned}
& \mathcal{V}_{0}=\left\{(e, \ell) \in \mathcal{V}:\left.e\right|_{\gamma}=0 \text { et }\left.\ell_{\Gamma}\right|_{\gamma}=0\right\}, \\
& \mathcal{N}_{0}=\left\{(e, \ell) \in \mathcal{V}_{0}: \varepsilon^{0}(e, \ell)=\varepsilon^{1}(e, \ell)=0\right\} ;
\end{aligned}
$$

lorsque, en plus, $\omega$ est connexe et $\gamma_{0}$ est une partie de $\gamma$ de $\omega$ de capacité $(N-1)$ non nulle

$$
\begin{aligned}
& \mathcal{V}_{\gamma_{0}}=\left\{(e, \ell) \in \mathcal{V}:\left.e\right|_{\gamma_{0}}=0 \text { et }\left.\ell_{\Gamma}\right|_{\gamma_{0}}=0\right\}, \\
& \mathcal{N}_{\gamma_{0}}=\left\{(e, \ell) \in \mathcal{V}_{\gamma_{0}}: \varepsilon^{0}(e, \ell)=\varepsilon^{1}(e, \ell)=0\right\} .
\end{aligned}
$$

Dans les deux cas $q(e, \ell)$ est défine par

$$
q(e, \ell)^{2}=\left\|\varepsilon^{0}(e, \ell)\right\|^{2}+\left\|\varepsilon^{1}(e, \ell)\right\|^{2}
$$

est une norme pour ces espaces. On aura besoin des lemmes suivants.

Lemme 4. Soit $\omega$ un domaine borné ouvert dans $\Gamma$ de frontière lipschitzienne $\gamma$ vérifiant l'Hypothèse 3.2. Lorsque $h$ tend vers zero, il existe $c=c(h)>0$ tel que pour tout $(e, \ell) \in \mathcal{V}_{0}\left(\right.$ resp. $\mathcal{V}_{\gamma_{0}}$ avec $\omega$ connexe $)$

$$
\begin{aligned}
\int_{\omega} 2 h|e|^{2} & +2 \frac{h^{3}}{3}|\ell|^{2} d \Gamma \\
& \leq c^{2} \int_{\omega} 2 h\left\|\varepsilon^{0}(e, \ell)\right\|^{2}+2 \frac{h^{3}}{3}\left\|\varepsilon^{1}(e, \ell)\right\|^{2}+2 \frac{h^{5}}{5}\left\|\varepsilon^{2}(e, \ell)\right\|^{2} d \Gamma .
\end{aligned}
$$

De là il vient une inégalité de Poincaré globale sans $\varepsilon^{2}(e, \ell)$.

Théorème 5 (Inégalité de Poincaré globale). Sous l'Hypothèse 3.2 soit $\omega$ un domaine ouvert borné (resp. connexe) dans $\Gamma$ de frontière lipschitzienne $\gamma$.

(i) $\mathcal{N}_{0}=\{(0,0)\}\left(\right.$ resp. $\left.\mathcal{N}_{\gamma_{0}}=\{(0,0)\}\right)$ et

(ii) $q(e, \ell)$ est une norme dans $\mathcal{V}_{0}\left(\right.$ resp. $\left.\mathcal{V}_{\gamma_{0}}\right)$ et il existe une constante $c>0$ telle que

$$
\|e\|_{L^{2}(\omega)}+\|\ell\|_{L^{2}(\omega)} \leq c\left[\left\|\varepsilon^{0}(e, \ell)\right\|_{L^{2}(\omega)}+\left\|\varepsilon^{1}(e, \ell)\right\|_{L^{2}(\omega)}\right],
$$

et $\left\|\varepsilon^{0}(e, \ell)\right\|_{L^{2}(\omega)}+\left\|\varepsilon^{1}(e, \ell)\right\|_{L^{2}(\omega)}$ est une norme dans $\mathcal{V}_{0}$ (resp. $\left.\mathcal{V}_{\gamma_{0}}\right)$ qui est équivalente à la norme $H^{1} \times H^{1}$. 


\subsection{Energie de déformation et travail des forces extérieures}

L'équivalence des conditions $\varepsilon(V) \circ T=0$ et $\varepsilon^{0}=\varepsilon^{1}=0$, et la caractérisation de l'espace $\mathcal{V}$ en fonction des deux tenseurs suggèrent l'utilisation d'une approximation du premier ordre du tenseur linéarisé des déformations.

Hypothèse 5.2. Le tenseur linéarisé des déformations $\varepsilon(V)$ sera approché par un développement du premier ordre (en $z$ )

$$
\tilde{\varepsilon}(V) \circ T_{z} \stackrel{\text { def }}{=} \varepsilon^{0}(e, \ell)+\varepsilon^{1}(e, \ell) z .
$$

Ceci confirme l'intérêt du modèle $P(1,1)$. On reprend le calcul de l'énergie de déformation plus le travail des forces externes dans le cas statique pour l'approximation $P(1,1)$. Dans le cas dynamique des petites vibrations on y soustraira l'énergie cinétique et l'on caractérisera les points d'équilibre. Par forces externes il faut comprendre ici l'effet combiné des forces et des moments appliqués à la structure. Pour simplifier on prend des forces distribuées dans $S_{h}(\omega)$, mais des cas plus généraux peuvent être envisagés.

De façon générique, l'énergie de déformation $\mathcal{P}$ et le travail des forces externes $\mathcal{W}$ sont donnés par

$$
\mathcal{P}=\frac{1}{2} \int_{S_{h}(\omega)} \sigma \cdot \tilde{\varepsilon}(V) d x, \quad \mathcal{W}=\int_{S_{h}(\omega)}[F \cdot V+(m \circ p) \cdot(\ell \circ p)] d x .
$$

Supposonst que $F$ dans $S_{h}(\omega)$ est de la forme

$$
F=f^{0} \circ p+b f^{1} \circ p, \quad f^{0}, f^{1} \in L^{2}(\omega)^{N},
$$

que $m \in L^{2}(\omega)^{N}$ et que le matériau obéit à une loi de comportement symétrique

$$
\sigma=C^{-1} \tilde{\varepsilon}(V)
$$

Alors

$$
\sigma \circ T_{z} \cdot \tilde{\varepsilon}(V) \circ T_{z}=C^{-1} \tilde{\varepsilon}(V) \circ T_{z} \cdot \tilde{\varepsilon}(V) \circ T_{z} .
$$

On utilise maintenant les expressions $V \circ T_{z}=e+z \ell, \tilde{\varepsilon}(V) \circ T_{z}=\varepsilon^{0}+z \varepsilon^{1}$, et la décomposition de la mesure de Federer le long des ensembles de niveau de $b$. De (51) et (53)

$$
\begin{aligned}
\mathcal{P} & =\frac{1}{2} \int_{-h}^{h} d z \int_{\omega} d \Gamma\left[C^{-1} \tilde{\varepsilon}(V) \circ T_{z} \cdot \tilde{\varepsilon}(V) \circ T_{z}\right] j_{z}, \\
\mathcal{W} & =\int_{-h}^{h} d z \int_{\omega} d \Gamma\left[F \circ T_{z} \cdot V \circ T_{z}+m \cdot\left(\ell \circ p \circ T_{z}\right)\right] j_{z} .
\end{aligned}
$$

En utilisant l'identité (54)

$$
\begin{gathered}
\sigma(V) \circ T_{z} \cdot \tilde{\varepsilon}(V) \circ T_{z} j_{z}=\sum_{i, j=0}^{1} z^{i+j} j_{z}\left[C^{-1} \varepsilon^{i} \cdot \varepsilon^{j}\right]=\sum_{n=0}^{2} z^{n} j_{z} A_{n}, \\
A_{n} \stackrel{\text { def }}{=} \sum_{\substack{i=0 \\
0 \leq n-i \leq 1}}^{1}\left[C^{-1} \varepsilon^{i} \cdots \varepsilon^{n-i}\right] .
\end{gathered}
$$

De façon analogue pour l'intégrande de (56)

$$
\begin{aligned}
(F \cdot V) \circ T_{z} & =m \cdot \ell+\left(f^{0}+z f^{1}\right) \cdot(e+z \ell) \\
& =f^{0} \cdot e+m \cdot \ell+z\left(f^{1} \cdot e+f^{0} \cdot \ell\right)+z^{2} f^{1} \cdot \ell .
\end{aligned}
$$


Les expressions finales de $\mathcal{P}$ et de $\mathcal{W}$ deviennent

$$
\begin{aligned}
\mathcal{P} & =\frac{1}{2} \int_{\omega} \int_{-h}^{h} \sum_{n=0}^{2} z^{n} j_{z} d z A_{n} d \Gamma=\frac{1}{2} \int_{\omega} \sum_{n=0}^{2} \alpha_{n}(h) A_{n} d \Gamma, \\
\mathcal{W} & =\int_{-h}^{h} d z \int_{\omega} d \Gamma\left[m \cdot \ell+\left(f^{0}+z f^{1}\right) \cdot(e+z \ell)\right] j_{z} \\
& =\int_{\omega} \alpha_{0}(h)\left(f^{0} \cdot e+m \cdot \ell\right)+\alpha_{1}(h)\left(f^{1} \cdot e+f^{0} \cdot \ell\right)+\alpha_{2}(h) f^{1} \cdot \ell d \Gamma .
\end{aligned}
$$

\subsection{Modèle linéaire statique}

5.5.1. La forme bilinéaire. Les espaces $\mathcal{H}, \mathcal{V}$, et $\mathcal{N}$, et leurs normes et semi-normes associées sont définis par (36) à (40). On introduit maintenant l'opérateur $A: \mathcal{V} \rightarrow \mathcal{V}^{\prime}$ associé à $\mathcal{P}:$ pour tous $(e, \ell)$ et $(\bar{e}, \bar{\ell})$ dans $\mathcal{V}$ et $\varepsilon^{i}=\varepsilon^{i}(e, \ell), \bar{\varepsilon}^{i}=\varepsilon^{i}(\bar{\epsilon}, \bar{\ell})$,

$$
\begin{gathered}
\langle A(e, \ell),(\bar{\epsilon}, \bar{\ell})\rangle_{\mathcal{V}} \stackrel{\text { def }}{=} \sum_{n=0}^{2} \int_{\omega} \alpha_{n}(h) a_{n}((e, \ell),(\bar{\epsilon}, \bar{\ell})) d \Gamma, \\
a_{n}((e, \ell),(\bar{\epsilon}, \bar{\ell})) \stackrel{\text { def }}{=} \sum_{\substack{i=0 \\
0 \leq n-i \leq 1}}^{1}\left(C^{-1} \varepsilon^{i}\right) \cdot \cdot \bar{\varepsilon}^{n-i} .
\end{gathered}
$$

Il est à noter que $A_{n}=a_{n}((e, \ell),(e, \ell)), 0 \leq n \leq 2$. On remarquera ici qu'il n'est pas nécessaire de passer par la minimisation de $\mathcal{P}-\mathcal{W}$ et donc pas nécessaire de supposer que $C$ est symétrique. Il suffit d'utiliser le principe des travaux virtuels et Lax-Milgram. De la même façon on définit l'opérateur linéaire continu associé à $\mathcal{W}$ : pour tous $(e, \ell)$ et $(\bar{e}, \bar{\ell})$ dans $\mathcal{V}$,

$$
\begin{gathered}
\mathcal{B}: U \stackrel{\text { def }}{=} L^{2}(\omega)^{N} \times L^{2}(\omega)^{N} \rightarrow \mathcal{H}^{\prime}, \\
\langle\mathcal{B}(f, m),(e, \ell)\rangle_{\mathcal{H}} \\
\stackrel{\text { def }}{=} \int_{\omega} \alpha_{0}\left(f^{0} \cdot e+m \cdot \ell\right)+\alpha_{1}\left(f^{1} \cdot e+f^{0} \cdot \ell\right)+\alpha_{2} f^{1} \cdot \ell d \Gamma .
\end{gathered}
$$

Lemme 6 ([28]). Sous les Hypothèses 3.1 et 3.2, il existe $\bar{h}>0$ et $\alpha>0$ tel que pour tout $0<h<\bar{h}$ et tout $(e, \ell) \in \mathcal{V}$

$$
\langle A(e, \ell),(e, \ell)\rangle_{\mathcal{V}} \geq 2 \mu h \alpha \sum_{n=0}^{1} h^{2 n}\left\|\varepsilon^{n}(e, \ell)\right\|^{2} .
$$

Si de plus $C$ est symétrique, alors l'opérateur $A$ est symétrique

$$
\forall(e, \ell),(\bar{e}, \bar{\ell}), \quad\langle A(e, \ell),(\bar{e}, \bar{\ell})\rangle_{\mathcal{V}}=\langle A(\bar{e}, \bar{\ell}),(e, \ell)\rangle_{\mathcal{V}} .
$$

5.5.2. Conditions homogènes de Neumann et Coque sans fronTière. Si les éléments du dual $\mathcal{H}^{\prime}$ de $\mathcal{H}$ sont identifiés avec ceux de $\mathcal{H}$, alors par le lemme précédent l'opérateur $A$ est $\mathcal{V}-\mathcal{H}$ coercif, c'est-à-dire

$$
\exists \alpha>0 \text { et } \beta \in \mathbb{R} \text { tels que }\langle A(e, \ell),(e, \ell)\rangle_{\mathcal{V}}+\beta\|(e, \ell)\|_{\mathcal{H}} \geq \alpha\|(e, \ell)\|_{\mathcal{V}}^{2} .
$$

Si $\omega=\Gamma$, le domaine $\omega$ n'a pas de frontière; si $\omega$ possède une frontière $\gamma$, on a la condition homogène de Neumann. Les deux cas sont couverts par le théorème suivant. 
Théorìme 7 . Sous les Hypothèses 3.1 et 3.2 soit $\bar{h}>0$ comme dans le Lemme $6,0<h \leq \bar{h}$. On fait l'hypothèse que la condition suivante est vérifiée pour tout $(e, \ell) \in \mathcal{N}$

$$
\int_{\omega} \alpha_{0}\left(f^{0} \cdot e+m \cdot \ell\right)+\alpha_{1}\left(f^{1} \cdot e+f^{0} \cdot \ell\right)+\alpha_{2} f^{1} \cdot \ell d \Gamma=0 .
$$

Alors pour tout $h, 0<h \leq \bar{h}$, il existe une solution unique $(\hat{e}, \hat{\ell}) \in \mathcal{V} / \mathcal{N} \grave{a}$ l'équation variationnelle: pour tout $(e, \ell) \in \mathcal{V}$

$$
\langle A(\hat{e}, \hat{\ell}),(e, \ell)\rangle_{\mathcal{V}}+\langle\mathcal{B}(f, m),(e, \ell)\rangle_{\mathcal{H}}=0 .
$$

5.5.3. Conditions homogines de Dirichlet. Pour une coque avec frontière et des conditions homogènes de type Dirichlet, les résultats sont analogues et se démontrent de la même façon que pour le Théorème 7 en utilisant l'inégalité de Poincaré globale du Théorème 5.

Théorìme 8. Soit $\bar{h}>0$ tel que spécifié au Lemme 6 et $h, 0<h \leq \bar{h}$. Il existe une solution unique $(\hat{e}, \hat{\ell}) \in \mathcal{V}_{0}$ à l'équation variationnelle : pour tout $(e, \ell) \in \mathcal{V}_{0}$

$$
\langle A(\hat{e}, \hat{\ell}),(e, \ell)\rangle_{\mathcal{V}}+\langle\mathcal{B}(f, m),(e, \ell)\rangle_{\mathcal{H}}=0 .
$$

Les mêmes résultats demeurent vrais pour l'espace $\mathcal{V}_{\gamma_{0}}$ lorsque $\omega$ est connexe et $\gamma_{0} \subset \gamma$ est de $(N-1)$-capacité non nulle.

Il est important de remarquer qu'il n'y a pas de condition frontière sur la composante normale $\ell_{n}$ de $\ell$ dans $\mathcal{V}_{0}$ et $\mathcal{V}_{\gamma_{0}}$.

\subsection{Le modèle linéatre dynamique}

5.6.1. L'énergie cinétique. Pour obtenir le modèle linéaire dynamique, on suppose que la transformation $C$ de la loi de comportement est symétrique et que les fonctions vectorielles $V(x, t), e(x, t)$, et $\ell(x, t)$ dependent du temps $t$. Étant données $V^{\prime}, \epsilon^{\prime}$, et $\ell^{\prime}$ les dérivées par rapport a 1 temps $t$, l'énergie cinétique est donnée par l'expression

$$
\begin{aligned}
\mathcal{K} & =\int_{S_{h}(\omega)} \frac{1}{2} \rho\left|V^{\prime}\right|^{2} d x=\int_{S_{h}(\omega)} \frac{1}{2} \rho\left|\ell^{\prime} \circ p+b \ell^{\prime} \circ p\right|^{2} d x \\
& =\frac{1}{2} \rho \int_{\omega} \alpha_{0}(h)\left|\ell^{\prime}\right|^{2}+\alpha_{1}(h) 2 e^{\prime} \cdot \ell^{\prime}+\alpha_{2}(h)\left|\ell^{\prime}\right|^{2} d \Gamma,
\end{aligned}
$$

où $\rho$ est la densité volumique du matériau. On définit $\mathcal{M}: \mathcal{H} \rightarrow \mathcal{H}^{\prime}$ :

$$
\langle\mathcal{M}(e, \ell),(\bar{\ell}, \bar{\ell})\rangle_{\mathcal{H}} \stackrel{\text { def }}{=} \rho \int_{\omega} \alpha_{0}(h) e \cdot \bar{\epsilon}+\alpha_{1}(h)[e \cdot \bar{\ell}+\bar{\ell} \cdot \ell]+\alpha_{2}(h) \ell \cdot \bar{\ell} d \Gamma .
$$

Pour $h>0$ suffisamment petit, $\mathcal{M}$ est symétrique, positif et inversible.

5.6.2. Le modèle dynamique. On identifie les éléments du dual $\mathcal{H}^{\prime}$ de $\mathcal{H}$ avec ceux de $\mathcal{H}$, on note par $\Lambda: \mathcal{H}^{\prime} \rightarrow \mathcal{H}$ l'isomorphisme canonique correspondant et l'on définit les opérateurs linéaires bornés

$$
M=\Lambda \mathcal{M}: \mathcal{H} \rightarrow \mathcal{H}, \quad B=\Lambda \mathcal{B}: U \rightarrow \mathcal{H}
$$

Pour $h>0$ suffisamment petit, $M$ est un opérateur linéaire, inversible, continu et symétrique. Un point stationnaire $(e, \ell)$ de l'énergie totale $\mathcal{P}+$ 
$\mathcal{W}-\mathcal{K}$ vérifie l'équation dynamique du second ordre

$$
\frac{d^{2}}{d t^{2}}\left(M\left[\begin{array}{l}
e(t) \\
\ell(t)
\end{array}\right],\left[\begin{array}{l}
\varphi \\
\psi
\end{array}\right]\right)_{\mathcal{H}}+\left\langle A\left[\begin{array}{l}
e(t) \\
\ell(t)
\end{array}\right],\left[\begin{array}{l}
\varphi \\
\psi
\end{array}\right]\right\rangle_{\mathcal{V}}+\left(B\left[\begin{array}{c}
f(t) \\
m(t)
\end{array}\right],\left[\begin{array}{l}
\varphi \\
\psi
\end{array}\right]\right)_{\mathcal{H}}=0
$$

pour tout $(\varphi, \psi) \in \mathcal{V}$. Les théorèmes habituels d'existence et d'unicité s'appliquent alors (cf. [59], Thm 1.1 et Rem. 1.3, p. 294]).

ThÉORÈme 9. Supposons que les Hypothèses 3.1 et 3.2 soient vérifiées avec $C$ symétrique. Soit $0<h \leq \bar{h}$, pour $\bar{h}>0$ tel que spécifié au Lemme 6 .

(i) (Coque sans frontière ou conditions homogènes de Neumann) Il existe une solution unique

$$
(e, \ell) \in C^{1}([0, T] ; \mathcal{H}) \cap C([0, T] ; \mathcal{V})
$$

à l'équation (72) vérifiant les conditions initiales

$(\epsilon(0), \ell(0))=\left(e_{0}, \ell_{0}\right)$ dans $\mathcal{V}, \quad\left(e^{\prime}(0), \ell^{\prime}(0)\right)=\left(e_{1}, \ell_{1}\right)$ dans $\mathcal{H}$.

(ii) (Conditions homogènes de Dirichlet) Il existe une solution unique

$$
(e, \ell) \in C^{1}([0, T] ; \mathcal{H}) \cap C\left([0, T] ; \mathcal{V}_{0}\right)
$$

à l'équation (72) vérifiant les conditions initiales

$(e(0), \ell(0))=\left(e_{0}, \ell_{0}\right)$ dans $\mathcal{V}_{0}, \quad\left(e^{\prime}(0), \ell^{\prime}(0)\right)=\left(e_{1}, \ell_{1}\right)$ dans $\mathcal{H}$.

Les mêmes résultats sont vrais pour $\mathcal{V}_{\gamma_{0}}$.

\section{LE MODÈLE ASYMPTOTIQUE $P(1,0)$}

Le modèle $P(1,0)$ correspond à l'équation variationnelle obtenue en ne gardant que le coefficient de $h$ dans la seconde expression (27) soit

$$
\begin{gathered}
\int_{\omega} C^{-1} \varepsilon^{0}(v) \cdots \varepsilon^{0}(\bar{v})-f^{0} \cdot \bar{v}^{0} d \Gamma=0, \quad \forall \bar{v}^{0}, \bar{v}^{1}, \\
\varepsilon^{0}(v) \stackrel{\text { def }}{=} \frac{1}{2}\left[v^{1 *} n+n^{*} v^{1}\right]+\varepsilon_{\Gamma}\left(v^{0}\right) .
\end{gathered}
$$

Cela donne deux équations variationnelles pour $v^{0}$ et $v^{1}$

$$
\begin{gathered}
\int_{\omega}\left\{C^{-1} \varepsilon^{0}(v) \cdots \frac{1}{2}\left[\bar{v}^{1 *} n+n^{*} \bar{v}^{1}\right]\right\} d \Gamma=0, \quad \forall \bar{v}^{1}, \\
\int_{\omega}\left\{C^{-1} \varepsilon^{0}(v) \cdots \varepsilon_{\Gamma}\left(\bar{v}^{0}\right)-f^{0} \cdot \bar{v}^{0}\right\} d \Gamma=0, \quad \forall \bar{v}^{0} .
\end{gathered}
$$

En décomposant les tenseurs en leur partie tangente et leur composante normale le long de $\omega$, il vient

$$
\left[C^{-1} \varepsilon^{0}(v)\right] n=0 \text { et } \int_{\omega}\left\{\left[C^{-1} \varepsilon^{0}(v)\right]^{P} \cdots \varepsilon_{\Gamma}^{P}\left(\bar{v}^{0}\right)-f^{0} \cdot \bar{v}^{0}\right\} d \Gamma=0, \quad \forall \bar{v}^{0},
$$

où $\varepsilon^{0 P}=P \varepsilon^{0} P$. Pour la loi de comportement $C^{-1} \varepsilon=2 \mu \varepsilon+\lambda \operatorname{tr} \varepsilon I$

$$
\left[C^{-1} \varepsilon^{0}(v)\right] n=2 \mu\left[\varepsilon_{\Gamma}\left(v_{0}\right) n+\frac{1}{2} v_{\Gamma}^{1}\right]+\left[\lambda \operatorname{tr} \varepsilon_{\Gamma}\left(v_{0}\right)+(2 \mu+\lambda) v_{n}^{1}\right] n
$$


et la condition $\left[C^{-1} \varepsilon^{0}(v)\right] n=0$ donne la composante normale et la partie tangentielle de $v^{1}$ en fonction de $v^{0}$

$$
\begin{gathered}
v_{n}^{1}=v^{1} \cdot n=-\frac{\lambda}{2 \mu+\lambda} \operatorname{div}_{\Gamma}\left(v^{0}\right)=-\frac{\lambda}{2 \mu+\lambda}\left[H v_{n}^{0}+\operatorname{div}_{\Gamma}\left(v_{\Gamma}^{0}\right)\right] \\
v_{\Gamma}^{1}=-2 \varepsilon_{\Gamma}\left(v^{0}\right) n=-\nabla_{\Gamma}\left(v_{n}^{0}\right)+D^{2} b v_{\Gamma}^{0} \text { (condition de Love-Kirchhoff). }
\end{gathered}
$$

La substitution de l'expression de $v^{1}$ dans

$$
\left[C^{-1} \varepsilon^{0}(v)\right]^{P}=2 \mu \varepsilon_{\Gamma}^{P}\left(v^{0}\right) \cdots \varepsilon_{\Gamma}^{P}\left(\bar{v}^{0}\right)+\frac{2 \mu \lambda}{2 \mu+\lambda} \operatorname{tr} \varepsilon_{\Gamma}^{P}\left(v^{0}\right) \operatorname{tr} \varepsilon_{\Gamma}^{P}\left(\bar{v}^{0}\right)
$$

donne la seconde équation pour $v^{0}$ : pour tout $\bar{v}^{0}$

$$
\begin{aligned}
& {\left[C^{-1} \varepsilon^{0}(v)\right]^{P} \cdots \varepsilon_{\Gamma}^{P}\left(\bar{v}^{0}\right)=2 \mu \varepsilon_{\Gamma}^{P}\left(v^{0}\right) \cdots \varepsilon_{\Gamma}^{P}\left(\bar{v}^{0}\right)+\frac{2 \mu \lambda}{2 \mu+\lambda} \operatorname{tr} \varepsilon_{\Gamma}^{P}\left(v^{0}\right) \operatorname{tr} \varepsilon_{\Gamma}^{P}\left(\bar{v}^{0}\right)} \\
& \int_{\omega} 2 \mu \varepsilon_{\Gamma}^{P}\left(v^{0}\right) \cdots \varepsilon_{\Gamma}^{P}\left(\bar{v}^{0}\right)+\frac{2 \mu \lambda}{2 \mu+\lambda} \operatorname{tr} \varepsilon_{\Gamma}^{P}\left(v^{0}\right) \operatorname{tr} \varepsilon_{\Gamma}^{P}\left(\bar{v}^{0}\right)-f^{0} \cdot \bar{v}^{0} d \Gamma=0 .
\end{aligned}
$$

C'est précisément l'équation asymptotique de coques membranaires avec les bons coefficients. Il est à noter que l'on obtient simultanément $v^{0}$ et $v^{1}$.

L'existence de solutions $\left(v_{\Gamma}^{0}, v_{n}^{0}\right)$ dans $H_{0}^{1}(\omega)^{3} \times L^{2}(\omega)$ à l'équation (79) à été étudiée dans [20] et Ciarlet et Lods [14] [17] ont établi l'unicité de la solution sous l'hypothèse d'ellipticité uniforme de la surface moyenne

$$
\exists \nu>0 \text { tel que } \forall \xi \in \mathbb{R}^{N}, \quad D^{2} b(X) \xi_{\Gamma} \cdot \xi_{\Gamma} \geq \nu\left|\xi_{\Gamma}\right|^{2} .
$$

Ceci veut dire que $\omega$ est un domaine contenu dans la frontière $\Gamma$ d'un sousensemble strictement convexe de $\mathbb{R}^{N}$.

On introduit les espaces quotients

$$
\begin{gathered}
\mathcal{V}^{0} / \operatorname{ker} \varepsilon^{0}, \quad \mathcal{V}^{0}=H^{1}(\omega)^{N} \times L^{2}(\omega)^{N} \\
\operatorname{ker} \varepsilon^{0}=\left\{\left(v^{0}, v^{1}\right) \in \mathcal{V}^{0}: \varepsilon^{0}\left(v^{0}, v^{1}\right)=0\right\}
\end{gathered}
$$

pour la coque sans bords ou les conditions homogènes de Neumann et

$$
\begin{gathered}
\mathcal{V}_{\gamma_{0}}^{0} / \operatorname{ker} \varepsilon_{\gamma_{0}}^{0}, \mathcal{V}_{\gamma_{0}}^{0}=H_{\gamma_{0}}^{1}(\omega)^{N} \times L^{2}(\omega)^{N} \\
\operatorname{ker} \varepsilon_{\gamma_{0}}^{0}=\left\{\left(v^{0}, v^{1}\right) \in \mathcal{V}_{\gamma_{0}}^{0}: \varepsilon^{0}\left(v^{0}, v^{1}\right)=0\right\}
\end{gathered}
$$

pour les conditions homogènes de Dirichlet (même chose pour Dirichlet sur tout $\gamma$ en remplaçant $\gamma_{0}$ par $\gamma$ ). Il est facile de vérifier que

$$
\begin{aligned}
& \operatorname{ker} \varepsilon^{0}=\left\{\left(v^{0}, v^{1}\right) \in H^{1}(\omega)^{N} \times L^{2}(\omega)^{N}:\right. \\
&\left.v_{n}^{1}=0, v_{\Gamma}^{1}=-2 \varepsilon_{\Gamma}\left(v^{0}\right) n, \varepsilon_{\Gamma}^{P}\left(v^{0}\right)=0\right\}, \\
& \operatorname{ker} \varepsilon_{\gamma_{0}}^{0}=\left\{\left(v^{0}, v^{1}\right) \in H_{\gamma_{0}}^{1}(\omega)^{N} \times L^{2}(\omega)^{N}:\right. \\
&\left.v_{n}^{1}=0, v_{\Gamma}^{1}=-2 \varepsilon_{\Gamma}\left(v^{0}\right) n, \varepsilon_{\Gamma}^{P}\left(v^{0}\right)=0\right\}
\end{aligned}
$$

et que puisque $v^{1}$ est donné explicitement en terme de $v^{0}$, les éléments du noyau sont complètement caractérisés en terme du noyau de $\varepsilon_{\Gamma}^{P}$

$$
\begin{aligned}
\operatorname{ker} \varepsilon_{\Gamma}^{P} & =\left\{v^{0} \in H^{1}(\omega)^{N}: \varepsilon_{\Gamma}^{P}\left(v^{0}\right)=0\right\} \\
\operatorname{ker}_{\gamma_{0}} \varepsilon_{\Gamma}^{P} & =\left\{v^{0} \in H_{\gamma_{0}}^{1}(\omega)^{N}: \varepsilon_{\Gamma}^{P}\left(v^{0}\right)=0\right\} .
\end{aligned}
$$

Théorème 10. Sous les Hypothèses 3.1 et 3.2 soit $\omega$ un domaine ouvert borné de frontière lipschitzienne $\gamma$ dans $\Gamma$. 
(i) Étant donnée $f^{0} \in L^{2}(\omega)^{N}$ vérifiant la condition

$$
\exists c>0, \forall \bar{v}^{0}, \quad\left|\int_{\omega} f^{0} \cdot \bar{v}^{0} d \Gamma\right| \leq c\left\|\varepsilon_{\Gamma}^{P}\left(\bar{v}^{0}\right)\right\|_{L^{2}(\omega)},
$$

l'équation variationnelle (77) possède une solution unique $\left(v^{0}, v^{1}\right)$ dans le complété $E^{0}$ de l'espace $\mathcal{V}^{0} /$ ker $\varepsilon^{0}$ par rapport à la norme $\left\|\varepsilon^{0}\left(v^{0}, v^{1}\right)\right\|$.

(ii) Soit $\gamma_{0}$ une partie $\gamma$ de $(N-1)$-capacité strictement positive (avec $\omega$ connexe si $\left.\gamma_{0} \neq \gamma\right)$. Étant donné $f^{0} \in L^{2}(\omega)^{N}$ tel que

$$
\exists c>0, \forall \bar{v}^{0}, \quad\left|\int_{\omega} f^{0} \cdot \bar{v}^{0} d \Gamma\right| \leq c\left\|\varepsilon_{\Gamma}^{P}\left(\bar{v}^{0}\right)\right\|_{L^{2}(\omega)},
$$

l'équation (77) admet une solution unique $\left(v^{0}, v^{1}\right)$ dans le complété $E_{\gamma_{0}}^{0}$ de l'espace $\mathcal{V}_{\gamma_{0}}^{0} / \operatorname{ker}_{\gamma_{0}} \varepsilon^{0}$ par rapport à la norme $\left\|\varepsilon^{0}\left(v^{0}, v^{1}\right)\right\|$.

\section{Convergence de $P(1,1)$ vers $P(1,0)$}

On aura besoin du théorème suivant.

Théorème 11. Soit $\omega$ un domaine ouvert borné dans $\Gamma$ vérifiant l'Hypothèse 3.2.

(i) On définit l'espace

$$
H_{n}^{1}\left(S_{h}(\omega)\right)^{N} \stackrel{\text { def }}{=}\left\{F \in L^{2}\left(S_{h}(\omega)\right)^{N}: D F \nabla b \in L^{2}\left(S_{h}(\omega)\right)^{N}\right\} .
$$

L'application

$$
F \mapsto F \circ T: H_{n}^{1}\left(S_{h}(\omega)\right)^{N} \rightarrow H^{1}\left(-h, h ; L^{2}(\omega)^{N}\right)
$$

est une bijection linéaire continue.

(ii) L'application

$$
F \mapsto F \circ T: H^{1}\left(S_{h}(\omega)\right)^{N} \rightarrow H^{1}\left(-h, h ; L^{2}(\omega)^{N}\right) \cap L^{2}\left(-h, h ; H^{1}(\omega)^{N}\right)
$$

est une bijection linéaire continue. De plus

$$
\begin{gathered}
(D F \nabla b) \circ T=\frac{\partial f}{\partial z}, \quad(D F P) \circ T=D_{\Gamma} f\left[I+z D^{2} b\right]^{-1} \\
D F \circ T=\frac{\partial f}{\partial z}{ }^{*} n+D_{\Gamma} f\left[I+z D^{2} b\right]^{-1}=\left[\frac{\partial f}{\partial z}{ }^{*} n+D_{\Gamma} f\right]\left[I+z D^{2} b\right]^{-1} .
\end{gathered}
$$

En fait

$$
H^{1}(\omega \times]-h, h[)^{N}=H^{1}\left(-h, h ; L^{2}(\omega)^{N}\right) \cap L^{2}\left(-h, h ; H^{1}(\omega)^{N}\right) .
$$

(iii) Étant donné $F \in H_{n}^{1}\left(S_{h}(\omega)\right)^{N}$ et $f=F \circ T$ l'application

$$
F \mapsto f(\cdot, 0): H_{n}^{1}\left(S_{h}(\omega)\right)^{N} \rightarrow L^{2}(\omega)^{N}
$$

est bien définie, linéaire et il existe une constante $c$, independante de $h$, telle que lorsque $h$ tend vers zéro

$$
\begin{aligned}
& \|f-f(\cdot, 0)\|_{L^{2}(\omega \times]-h, h[)} \leq c h\|D F \nabla b\|_{L^{2}\left(S_{h}(\omega)\right)} \\
& \|f(\cdot, 0)\|_{L^{2}(\omega)} \leq \frac{c}{h^{1 / 2}}\left[\|F\|_{L^{2}\left(S_{h}(\omega)\right)}+h\|D F \nabla b\|_{L^{2}\left(S_{h}(\omega)\right)}\right]
\end{aligned}
$$

et pour tout $V \in H_{n}^{1}\left(S_{h}(\omega)\right)^{N}$ et $v=V \circ T$

$$
\begin{aligned}
& \left|\int_{S_{h}(\omega)} F \cdot V-\frac{1}{\alpha_{0}(h) \circ T^{-1}} f(\cdot, 0) \circ p \cdot v(\cdot, 0) \circ p d x\right| \\
& \leq c h\|F\|_{H_{n}^{1}\left(S_{h}(\omega)\right)}\left[\|V\|_{L^{2}\left(S_{h}(\omega)\right)}+\|D V \nabla b\|_{L^{2}\left(S_{h}(\omega)\right)}\right] .
\end{aligned}
$$




\subsection{Coque sans frontière ou Conditions de Neumann}

À la lumière $\mathrm{d} u$ Théorème 11 on fait l'hypothèse suivante.

Hypothisse 7.1. Il existe $\bar{h}>0$ et une constante $c=c(\omega)>0$ tel que la fonction vectorielle $F$ apppartienne à $H_{n}^{1}\left(S_{\bar{h}}(\omega)\right)^{N}$ et que pour tout $h$, $0<h \leq \bar{h}$,

$$
\forall V \in \operatorname{ker} \varepsilon, \quad \int_{S_{h}(\omega)} F \cdot V d x=0
$$

et pour tous $\left(v^{0}, v^{1}\right) \in H^{1}(\omega)^{N} \times H^{1}(\omega)^{N}$

$$
\begin{aligned}
\left|\frac{1}{2 h} \int_{S_{h}(\omega)} F \cdot\left(v^{0} \circ p+b v^{1} \circ p\right) d x\right| \leq c\left\{\int_{\omega}[\right. & \left\|\varepsilon^{0}\left(v^{0}, v^{1}\right)\right\|^{2} \\
& \left.\left.+h^{2}\left\|\varepsilon^{1}\left(v^{0}, v^{1}\right)\right\|^{2}\right] d \Gamma\right\}^{\frac{1}{2}} .
\end{aligned}
$$

On définit $f^{0} \stackrel{\text { def }}{=} F \circ T(\cdot, 0) \in L^{2}(\omega)^{N}$.

La condition (91) est naturelle pour donner un sens aux solutions de l'équation variationnelle (18) dans l'espace quotient $H^{1}\left(S_{h}(\omega)\right)^{N} / \operatorname{ker} \varepsilon$ dans le cas de Neumann. Le cas de Dirichlet homogène utilise la même condition à la différence que la condition (19) sur le noyau disparaît et que l'espace quotient devient l'espace de Sobolev des fonctions vectorielles qui sont nulles sur la partie $\gamma_{0}$ de la frontière. Pour $V^{1}=v^{0} \circ p+b v^{1} \circ p$, on obtient un membre de gauche de la forme

$$
\begin{gathered}
\int_{S_{h}(\omega)} F \cdot V^{1} d x=2 h \int_{\omega} F_{h}^{0} \cdot v^{0}+F_{h}^{1} \cdot v^{1} d \Gamma \\
F_{h}^{0} \stackrel{\text { def }}{=} \frac{1}{2 h} \int_{-h}^{h} j_{z} F \circ T d z \text { et } F_{h}^{1} \stackrel{\text { def }}{=} \frac{1}{2 h} \int_{-h}^{h} j_{z} z F \circ T d z .
\end{gathered}
$$

En particulier 1'Hypothèse 7.1 est vérifiée pour $F=g \circ p$ avec $g \in L^{2}(\omega)^{N}$ tel que

$$
\exists c>0, \forall v^{0} \in H^{1}(\omega)^{N}, \quad\left|\int_{\omega} g \cdot v^{0} d \Gamma\right| \leq c\left\|\varepsilon_{\Gamma}^{P}\left(v^{0}\right)\right\|_{L^{2}(\omega)} .
$$

Dans ce cas

$$
F_{h}^{0}=\bar{\alpha}_{0}(h) g \text { et } F_{h}^{1}=\bar{\alpha}_{1}(h) g, \text { où } \bar{\alpha}_{i}(h) \stackrel{\text { def }}{=} \alpha_{i}(h) /(2 h) .
$$

Lemme 12. Supposons vérifiée l'Hypothèse 7.1.

(i) Lorsque $h>0$ tend vers zéro il existe $c>0$ tel que $\forall\left(v^{0}, v^{1}\right) \in$ $H^{1}(\omega)^{N} \times H^{1}(\omega)^{N}$

$$
\begin{gathered}
\left|\int_{\omega} F_{h}^{0} \cdot v^{0}+F_{h}^{1} \cdot v^{1} d \Gamma\right| \leq c\left\{\int_{\omega}\left\|\varepsilon^{0}\left(v^{0}, v^{1}\right)\right\|^{2}+h^{2}\left\|\varepsilon^{1}\left(v^{0}, v^{1}\right)\right\|^{2} d \Gamma\right\}^{\frac{1}{2}} \\
\forall\left(v^{0}, v^{1}\right) \in \operatorname{ker} \varepsilon^{0} \cap \operatorname{ker} \varepsilon^{1}, \quad \int_{\omega} F_{h}^{0} \cdot v^{0}+F_{h}^{1} \cdot v^{1} d \Gamma=0 .
\end{gathered}
$$

(ii) Pour tout $\left(v^{0}, v^{1}\right) \in H^{1}(\omega)^{N} \times L^{2}(\omega)^{N}$

$$
\begin{gathered}
\left|\int_{\omega} f^{0} \cdot v^{0} d \Gamma\right| \leq c\left\{\int_{\omega}\left\|\varepsilon^{0}\left(v^{0}, v^{1}\right)\right\|^{2} d \Gamma\right\}^{\frac{1}{2}} \\
\forall\left(v^{0}, v^{1}\right) \in \operatorname{ker} \varepsilon^{0}, \quad \int_{\omega} f^{0} \cdot v^{0} d \Gamma=0 .
\end{gathered}
$$


(iii) Lorsque $h>0$ tend vers zéro

$$
\begin{aligned}
& \int_{\omega}\left(F_{h}^{0}-f^{0}\right) \cdot v^{0}+F_{h}^{1} \cdot v^{1} d \Gamma=0, \quad \forall\left(v^{0}, v^{1}\right) \in \operatorname{ker} \varepsilon^{0} \cap \operatorname{ker} \varepsilon^{1} \\
& \text { et il existe } c>0 \text { tel que } \forall\left(v^{0}, v^{1}\right) \in H^{1}(\omega)^{N} \times H^{1}(\omega)^{N} \\
& \left|\int_{\omega}\left(F_{h}^{0}-f^{0}\right) \cdot v^{0}+F_{h}^{1} \cdot v^{1} d \Gamma\right| \\
& \leq c h^{1 / 2}\|F\|_{H_{n}^{1}\left(S_{h}(\omega)\right)}\left\{\left\|\varepsilon^{0}\left(v^{0}, v^{1}\right)\right\|_{L^{2}(\omega)}+\left\|\varepsilon^{1}\left(v^{0}, v^{1}\right)\right\|_{L^{2}(\omega)}\right\} .
\end{aligned}
$$

Soit $\left(v_{h}^{0}, v_{h}^{1}\right)$ la solution dans $H^{1}(\omega)^{N} \times H^{1}(\omega)^{N} \operatorname{modulo} \operatorname{ker} \varepsilon^{0} \cap \operatorname{ker} \varepsilon^{1}$ de l'équation variation nelle

$$
\int_{\omega} \sum_{i, j=0}^{1} \bar{\alpha}_{i+j}(h) C^{-1} \varepsilon^{i}\left(v_{h}^{0}, v_{h}^{1}\right) \cdots \varepsilon^{j}\left(\bar{v}^{0}, \bar{v}^{1}\right)-\sum_{i=0}^{1} F_{h}^{i} \cdot \bar{v}^{i} d \Gamma=0
$$

pour tous $\left(v^{0}, v^{1}\right) \in H^{1}(\omega)^{N} \times H^{1}(\omega)^{N}$. Par coercivité de $C^{-1}$ il existe $\alpha>0$ tel que

$$
\begin{aligned}
\alpha\left\{\left\|\varepsilon_{h}^{0}\right\|_{L^{2}}^{2}+h^{2}\left\|\varepsilon_{h}^{1}\right\|_{L^{2}}^{2}\right\} & \leq \sum_{i, j=0}^{1} \int_{\omega} \bar{\alpha}_{i+j}(h) C^{-1} \varepsilon_{h}^{i} \cdots \varepsilon_{h}^{j} d \Gamma \\
& =\int_{\omega} \sum_{i=0}^{1} F_{h}^{i} \cdot v_{h}^{i} d \Gamma \leq c\left\{\left\|\varepsilon_{h}^{0}\right\|_{L^{2}}^{2}+h^{2}\left\|\varepsilon_{h}^{1}\right\|_{L^{2}}^{2}\right\}^{\frac{1}{2}}
\end{aligned}
$$

pour $\varepsilon_{h}^{i} \stackrel{\text { def }}{=} \varepsilon^{i}\left(v_{h}^{0}, v_{h}^{1}\right)$, et $\bar{\varepsilon}^{i} \stackrel{\text { def }}{=} \varepsilon^{i}\left(\bar{v}^{0}, \bar{v}^{1}\right)$. Alors lorsque $h$ tend vers zéro

$$
\left\|\varepsilon^{0}\left(v_{h}^{0}, v_{h}^{1}\right)\right\|_{L^{2}}^{2}+h^{2}\left\|\varepsilon^{1}\left(v_{h}^{0}, v_{h}^{1}\right)\right\|_{L^{2}}^{2}=\left\|\varepsilon_{h}^{0}\right\|_{L^{2}}^{2}+h^{2}\left\|\varepsilon_{h}^{1}\right\|_{L^{2}}^{2} \leq c^{2} / \alpha^{2} .
$$

Donc $\varepsilon^{0}\left(v_{h}^{0}, v_{h}^{1}\right)$ et $h \varepsilon^{1}\left(v_{h}^{0}, v_{h}^{1}\right)$ sont bornés dans $L^{2}$. On peut montrer que $\left(v_{h}^{0}, v_{h}^{1}\right) \in\left(H^{1}(\omega)^{N} \times L^{2}(\omega)^{N}\right) /$ ker $\varepsilon^{0}$ et conclure que $\left(v_{h}^{0}, v_{h}^{1}\right)$ est borné puisque $\left\|\varepsilon^{0}\left(v^{0}, v^{1}\right)\right\|$ est une norme dans $H^{1}(\omega)^{N} \times L^{2}(\omega)^{N} / \operatorname{ker} \varepsilon^{0}$.

\section{LEMME 13. L'injection}

$$
\frac{H^{1}(\omega)^{N} \times H^{1}(\omega)^{N}}{\operatorname{ker} \varepsilon^{0} \cap \operatorname{ker} \varepsilon^{1}} \subset \frac{H^{1}(\omega)^{N} \times L^{2}(\omega)^{N}}{\operatorname{ker} \varepsilon^{0}} \subset E^{0}
$$

est continue et pour tout $\left(v^{0}, v^{1}\right) \in\left(H^{1}(\omega)^{N} \times H^{1}(\omega)^{N}\right) /\left(\operatorname{ker} \varepsilon^{0} \cap \operatorname{ker} \varepsilon^{1}\right)$.

À la lumière du Lemme 13 et $\mathrm{d} u$ fait que $\varepsilon^{0}\left(v_{h}^{0}, v_{h}^{1}\right)$ et $h \varepsilon^{1}\left(v_{h}^{0}, v_{h}^{1}\right)$ sont bornés, on a la convergence.

THÉORÈme 14. Sous les Hypothèses 3.1, 3.2 et 7.1 soit $\omega$ un domaine ouvert borné dans $\Gamma$. Lorsque $h$ tend vers zéro, la solution

$$
\left(v_{h}^{0}, v_{h}^{1}\right) \in\left(H^{1}(\omega)^{N} \times H^{1}(\omega)^{N}\right) /\left(\operatorname{ker} \varepsilon^{0} \cap \operatorname{ker} \varepsilon^{1}\right)
$$

de l'équation $(94)$ du modèle $P(1,1)$ converge vers la solution

$$
\left(\hat{v}^{0}, \hat{v}^{1}\right) \in E^{0}
$$

de l'équation (77) du modèle asymptotique $P(1,0)$ dans le sens suivant: lorsque $h$ tend vers zéro

$$
\int_{\omega} C^{-1} \varepsilon^{0}\left(v_{h}^{0}-\hat{v}^{0}, v_{h}^{1}-\hat{v}^{1}\right) \cdot \cdot \varepsilon^{0}\left(v_{h}^{0}-\hat{v}^{0}, v_{h}^{1}-\hat{v}^{1}\right) d \Gamma \rightarrow 0 .
$$




\subsection{Conditions de Dirichlet}

Pour les conditions homogènes de Dirichlet l'équation variationnelle (18) en dimension $N$ possède une solution unique dans l'espace $H_{\gamma_{0}}^{1}\left(S_{h}(\omega)\right)^{N}$ pour tout $F \in L^{2}\left(S_{h}(\omega)\right)^{N}$. En vertu de l'équivalence des normes ceci est équivalent à la condition : il existe $c=c\left(S_{h}(\omega)\right)>0$ tel que

$$
\forall V \in H_{\gamma_{0}}^{1}\left(S_{h}(\omega)\right)^{N}, \quad\left|\int_{S_{h}(\omega)} F \cdot V d x\right| \leq c\|\varepsilon(V)\|_{L^{2}\left(S_{h}(\omega)\right)} .
$$

On fait l'Hypothèse 7.1 et l'on procède comme dans le paragraphe précédent. Évidemment le Lemme 12 et le Théorème 11 demeurent vrais pour $\left(v^{0}, v^{1}\right) \in$ $H_{\gamma_{0}}^{1}(\omega)^{N} \times H_{\gamma_{0}}^{1}(\omega)^{N}$. Soit $\left(v_{h}^{0}, v_{h}^{1}\right)$ la solution dans $H_{\gamma_{0}}^{1}(\omega)^{N} \times H_{\gamma_{0}}^{1}(\omega)^{N}$ de l'équation variation nelle

$$
\left.\sum_{i, j=0}^{1} \int_{\omega} \bar{\alpha}_{i+j}(h)\left\{C^{-1} \varepsilon^{i}\left(v_{h}^{0}, v_{h}^{1}\right) \cdots \varepsilon^{j}\left(\bar{v}^{0}, \bar{v}^{1}\right)\right)\right\}-\sum_{i=0}^{1} F_{h}^{i} \cdot \bar{v}^{i} d \Gamma=0
$$

pour tout $\left(v^{0}, v^{1}\right) \in H_{\gamma_{0}}^{1}(\omega)^{N} \times H_{\gamma_{0}}^{1}(\omega)^{N}$. Par la même technique que précédemment on obtient aussi la convergence faible.

Théorème 15. Sous les Hypothèses 3.1, 3.2 et 7.1 soit $\omega$ un domaine ouvert borné de frontière lipschizienne $\gamma$ dans $\Gamma\left(\omega\right.$ connexe lorsque $\left.\gamma_{0} \neq \gamma\right)$. Lorsque $h$ tend vers zéro la solution

$$
\left(v_{h}^{0}, v_{h}^{1}\right) \in\left(H_{\gamma_{0}}^{1}(\omega)^{N} \times H_{\gamma_{0}}^{1}(\omega)^{N}\right) /\left(\operatorname{ker} \varepsilon^{0} \cap \operatorname{ker} \varepsilon^{1}\right)
$$

de l'équation (96) du modèle $P(1,1)$ converge vers la solution

$$
\left(\hat{v}^{0}, \hat{v}^{1}\right) \in E_{\gamma_{0}}^{0}
$$

du modèle asymptotique $P(1,0)$ : pour tout $\left(\bar{v}^{0}, \bar{v}^{1}\right) \in H_{\gamma_{0}}^{1}(\omega)^{N} \times L^{2}(\omega)^{N}$,

$$
\int_{\omega}\left\{C^{-1} \varepsilon^{0}\left(\hat{v}^{0}, \hat{v}^{1}\right) \cdot \varepsilon^{0}\left(\bar{v}^{0}, \bar{v}^{1}\right)-f^{0} \cdot \bar{v}^{0}\right\} d \Gamma=0
$$

dans le sens suivant: lorsque $h$ tend vers zéro

$$
\int_{\omega} \varepsilon^{0}\left(v_{h}^{0}-\hat{v}^{0}, v_{h}^{1}-\hat{v}^{1}\right) \cdot \varepsilon^{0}\left(v_{h}^{0}-\hat{v}^{0}, v_{h}^{1}-\hat{v}^{1}\right) d \Gamma \rightarrow 0
$$

\section{REFERENCES}

[1] E. Acerbi, G. Buttazzo, et D. Percivale, A variational definition for the strain energy of an elastic string, J. Elasticity 25 (1991), 137-148.

[2] S. M. Alessandrini, Some two-dimensional plate models: Derivation, asymptotic properties, and numerical approximation, Ph.D. thesis, Rutgers University, New Brunswick, New Jersey, 1991.

[3] S. M. Alessandrini, D. N. Arnold, R. S. Falk, et A. Madureira, Derivation and justification of plate models by variational methods, Proc. Plates and shells: From theory to practice, CRM Proc. Lecture Notes, Amer. Math. Soc., Providence, R.I. (à paraître).

[4] S. S. Antman, Nonlinear problems of elasticity, Springer-Verlag, New York, 1991.

[5] Th. Aubin, Nonlinear analysis on manifolds. Monge-Ampère equations, SpringerVerlag, Heidelberg, Berlin, 1982.

[6] M. Bernadou, Méthodes d'éléments finis pour les problèmes de coques minces, Masson, Paris, Milan, Barcelone, 1994.

[7] M. Bernadou et Ph. G. Ciarlet, Sur l'ellipticité du modèle linéaire de coques de W. T. Koiter, Computing Methods in Applied Sciences and engineering (R. Glowinski et J. L. Lions, eds.), Springer-Verlag, Berlin, Heidelberg, New York, 1976, pp. 89-136,

[8] M. Bernadou, Ph. G. Ciarlet, et B. Miara, Existence theorems for two-dimensional linear shell theories, J. Elasticity 34 (1994), 111-138. 
[9] F. Bourquin, Ph. G. Ciarlet, G. Geymonat, et A. Raoult, T-convergence et analyse asymptotique des plaques minces, C. R. Acad. Sci. Paris Sér. I Math. 315 (1992), $1017-1024$.

[10] F. Brezzi et M. Fortin, Mixed and hybrid finite element methods, Springer-Verlag, New York, Berlin, Heidelberg, 1991.

[11] C. Chen, Asymptotic convergence rates for the Kirchhoff plate model, Ph.D. Thesis, Penn. State, University Park, Pennsylvania, 1995.

[12] Ph. G. Ciarlet, Mathematical elasticity. I. Three-dimensional elasticity, Stud. Math. Appl., vol. 20, North-Holland Publishing Co., Amsterdam-New York, 1988.

[13] Plates and junctions in elastic multi-structures: An asymptotic analysis, Masson, Paris, Milano, Barcelona, Mexico, and Springer-Verlag, Berlin, New York, 1990.

[14] Ph. G. Ciarlet et V. Lods, Ellipticité des équations membranaires d'une coque uniformément elliptique, C. R. Acad. Sci. Paris Sér. I Math. 318 (1994), 195-200.

[15] Analyse asymptotique des coques linéairement élastiques. I. Coques membranaires, C. R. Acad. Sci. Paris Sér. I Math. 318 (1994), 863-868.

[16] Analyse asymptotique des coques linéairement élastiques. III. Une justification du modèle de Koiter, C. R. Acad. Sci. Paris Sér. I Math. 319 (1994), 299-304.

[17] On the ellipticity of linear membrane shell equations, Publication R 94010, Laboratoire d'analyse numérique, Univ. Pierre et Marie Curie, Paris, mai 1994.

[18] Ph. G. Ciarlet, V. Lods, and B. Miara, Analyse asymptotique des coques linéairement élastiques. II. Coques en flexion, C. R. Acad. Sci. Paris Sér. I Math. 319 (1994), $95-100$.

[19] Asymptotic analysis of linearly elastic shells. II. Justification of flexural ahell equations, Arch. Rational Mech. Anal., à paraître.

[20] $\mathrm{Ph}$. G. Ciarlet et E. Sanchez-Palencia, Un théorème d'existence et d'unicité pour les équations des coques membranaires, C. R. Acad. Sci. Paris Sér. I Math. 317 (1993), 801-805.

[21] M. Dauge, Complete asymptotics in thin elastic plates and optimal estimates for Kirchhoff-Love model, Preprint no. 95-06, Institut de Recherche Mathématique de Rennes, Université de Rennes, février 1995.

[22] M. Dauge et I. Gruais, Développement asymptotique d'ordre arbitraire pour une plaque élastique mince encastrée, C. R. Acad. Sc. Paris Sér. I Math. 321, (1995), 375-380.

[23] M. C. Delfour et J. Zhao, Intrinsic nonlinear models of shells for Saint VenantKirchhoff materials, CRM Report, Université de Montréal, avril 1996.

[24] M. C. Delfour et J.-P. Zolésio, Shape analysis via distance functions, J. Funct. Anal. 123 (1994), 129-201.

[25] Oriented distance function in shape analysis and optimization, Control and Optimal Design of Distributed Parameter Systems (J. Lagnese, D. L. Russell, L. White, eds.), pp. 39-72, Springer-Verlag, Berlin, Heidelberg, New York, Tokyo, 1994 .

[26] On a variational equation for thin shells, Control and Optimal Design of Distributed Parameter Systems, (J. Lagnese, D. L. Russell, L. White, eds.), SpringerVerlag, Berlin, Heidelberg, New York, Tokyo, 1994, pp. 25-37.

[27] A boundary differential equation for thin shells, J. Differential Equations 119 (1995), 426-449.

[28] Tangential differential equations for dynamical thin/shallow shells, J. Differential Equations 128 (1996), 125-167.

[29] Differential equations for linear shells: comparison between intrinsic and classical models, dans "Advances in the Mathematical Sciences - CRM's 25 years, (Luc Vinet, ed.), CRM Proc. Lecture Notes, Amer. Math. Soc., Providence, RI, à paraître.

[30] Shape analysis via distance functions: Local theory, CRM Report 2299, mars 1996, Université de Montréal.

[31] On the design and control of systems governed by differential equations on submanifolds, Control and Cybernet. 25 (1996), 497-514.

[32] Hidden boundary smoothness for some classes of differential equations on submanifolds, dans "Proc. 1996 Joint Summer Research Conference on Optimization Methods in PDE's", I. Lasiecka and S. Cox, Contemporary Mathematics, AMS Publications, Providence, R.I., à paraître.

[33] Convergence to the asymptotic model for linear thin shells, dans "Proc. 1996 Joint Summer Research Conference on Optimization Methods in PDE's", I. Lasiecka 
et S. Cox, Contemporary Mathematics, AMS Publications, Providence, R.I., à paraître.

[34] Intrinsic differential geometry and theory of thin shells, Lecture Notes, Scuola Normale Superiore, Pisa (Italy), août 1996.

[35] Ph. Destuynder, Sur la justification des modèles de plaques et de coques par les méthodes asymptotiques, Thèse de doctorat, Université Pierre et Marie Curie, 1980.

[36] Modélisation des coques minces élastiques, Masson, Paris, Milan, Barcelone, 1990.

[37] Une théorie asymptotique des plaques minces en elasticité linéaire, Masson, Paris, Milan, Barcelone, 1986.

[38] L. C. Evans et R. F. Gariepy, Measure theory and the properties of functions, Stud. Adv. Math., CRC Press, Boca Raton, FL, 1992.

[39] H. Federer, Hausdorff measure and Lebesgue area, Proc. Nat. Acad. Sci. U.S.A. 37 (1951), 90-94.

[40] Curvature measures, Trans. Amer. Math. Soc. 93 (1959), 418-419.

[41] H. Federer et W. H. Fleming, Normal and integral currents, Ann. of Math. (2) 72 (1960), 458-520.

[42] G. Fichera, Linear elliptic differential systems and eigenvalue problems, Lecture Notes in Math., vol. 8, Springer-Verlag, Berlin-New York, 1965.

[43] I. M. N. Figueiredo, Local existence and regularity of the solutions of the nonlinear thin shell model of Donnell-Mushtari-Vlasov, Appl. Anal. 36 (1990), 221-234.

[44] W. H. Fleming et R. Rishel, An integral formula for total gradient variation, Arch. Math. 11 (1960), 218-222.

[45] D. D. Fox, A. Raoult, et J. C. Simo, A justification of nonlinear properly invariant plate theories, Arch. Rational Mech. Anal. 124 (1993), 157-199.

[46] P. Germain, Mécanique. I-II, Ellipses éditeur, Paris, 1986.

[47] G. Geymonat, Sui problemi ai limiti per i sistemi lieari ellitici, Ann. Mat. Pura Appl. (4) 69 (1965), 207-284.

[48] D. Gilbarg et N. S. Trudinger, Elliptic partial differential equations of second order, Springer-Verlag, Berlin, Heidelberg, New York, Tokyo, 1983.

[49] A. L. Gol'denveizer, Theory of elastic thin shells, Pergamon Press, Oxford, 1961.

[50] A. E. Green, P. M. Naghdi, et W. L. Wainwright, A general theory of a Cosserat surface, Arch. Rational Mech. Anal. 20 (1965), 287-308.

[51] A. E. Green et W. Zerna, Theoretical elasticity, Oxford at the Clarendon Press, London, 1968.

[52] O. V. Guseva, On boundary problems for strongly elliptic systems, Dokl. Akad. Nauk USSR 102 (1955), 1069-1072.

[53] G. Kirchhoff, Vorlesungen über Mathematische Physik, Mechanik, Leipzig, 1876.

[54] W. T. Koiter, On the foundations of the linear theory of thin elastic shells. I, Proc. Konink. Nederl. Akad. Wetensch. B73 (1970), 169-195.

[55] On the nonlinear theory of thin elastic shells, Proc. Konink. Nederl. Akad. Wetensch. B69 (1966), 1-54.

[56] J. E. Lagnese et J.-L. Lions, Modelling, analysis and control of thin plates, RMA, Vol. 6, Masson, Paris, Milan, Barcelone, Mexico, 1988.

[57] H. Le Dret et A. Raoult, The nonlinear membrane model as variational limit of nonlinear three-dimensional elasticity, J. Math. Pures Appl. (9) 74 (1995), 549-578.

[58] The membrane shell model in nonlinear elasticity: A variational asymptotic derivation, J. Nonlinear Sci. 6 (1996), 59-84.

[59] J.-L. Lions, Contrôle optimal de systèmes gouvernés par des équations aux dérivées partielles, Dunod, Gauthier-Villars, Paris, 1968.

[60] K. H. Lo, R. M. Christensen, et E. M. Wu, A high-order theory of plate deformations, J. Appl. Mech. 46 (1977), 663-676.

[61] A. E. H. Love, The mathematical theory of elasticity, Cambridge University Press, 1934.

[62] C. Mardare, Modèles bi-dimensionnels de coques linéairement élastiques: Estimations de l'écart entre leurs solutions, C. R. Acad. Sci. Paris Sér. I Math. 322 (1996), 793796.

[63] Estimation d'erreur dans l'analyse asymptotique des coques linéairement élastiques, C. R. Acad. Sci. Paris Sér. I Math. 322 (1996), 895-898. 
[64] D. Morgenstern, Herleitung der Plattentheorie aus der dreidimensionalen Elastizitätstheorie, Arch. Rational Mech. Anal. 4 (1959), 145-152.

[65] P. M. Naghdi, Foundations of elastic shell theory, Progress in Solid Mechanics (I. N. Sueddon et R. Hill, eds.), vol. IV, North-Holland Publ. Co. Amsterdam, 1963, pp. 190.

[66] The theory of shells and plates, Handbuch der Physik, vol. VI a-2, SpringerVerlag, Berlin, 1972, pp. 425-640.

[67] J. Nečas, Les méthodes directes en théorie des équations elliptiques, Masson, Paris and Academia, Prague, 1967.

[68] V. V. Novozhilov, Thin shell theory, Wolters-Noordhoff Publishing, Groningen, 1970.

[69] J.-C. Paumier et A. Raoult, Asymptotic consistency of the polynomial approximation in the linearized plate theory. Application to the Reissner-Mindlin model, dans "Elasticity, Viscoelasticity and Optimal Control: Theoretical and Numerical Aspects", (Lyon, France, 1995), ESAIM Proccedings (à paraître).

[70] J. Sokolowski et J.-P. Zolésio, Introduction to shape optimization: Shape sensitivity analysis, Springer-Verlag, New York, Berlin, Heidelberg, 1992.

[71] R. Temam, Problèmes mathématiques en plasticité, Méthodes Mathématiques de l'Informatique, vol. 12, Gauthier-Villars, Paris, 1983.

[72] Monotone rearrangement of a function and the Grad-Mercier equation of plasmas physics, Recent Methods in Non Linear Analysis and Application (E. De Giorgi, E. Magenes, et U. Mosco, eds.), Pitagora Editrice, Bologna, 1979, pp.83-98.

[73] R. Valid, Mechanics of continuous media and analysis of structures, North-Holland Ser. Appl. Math. Mech. 26, North-Holland Publishing Co., Amsterdam-New York, 1981.

[74] The nonlinear theory of shells through variational principles, Wiley, Chichester, 1995.

[75] J. Zhao, Natural theory of nonlinear shells, Ph.D. Thesis, McGill University, April 1996.

[76] J.-P. Zolésio, Identification de domaines par déformation, thèse de doctorat d'état, Université de Nice, France, 1979.

[77] The material derivative (or speed) method for shape optimization, Optimization of Distributed Parameter Structures (Iowa City, Iowa, 1980) (E. J. Haug et J. Céa, eds.), vol. II, NATO Adv. Study Inst. Ser. E: Appl. Sci., vol. 50, Nijhoff, The Hague, 1981, pp. 1089-1151.

[78] Domain variational formulation for free boundary problems, Optimization of Distributed Parameter Structures (Iowa City, Iowa, 1980) (E. J. Haug et J. Céa, eds.), vol. II, NATO Adv. Study Inst. Ser. E: Appl. Sci., vol. 50, Nijhoff, The Hague, 1981, pp. 1152-1194.

Remerciements: Ces travaux ont été réalisés dans le cadre de la subvention de recherche A-8730 du Conseil National de Recherche en Sciences Naturelles et Génie et d'une subvention d'équipe du programme FCAR du Ministère de l'Éducation du Québec. L'auteur désire remercier A. Raoult (LMC, Université J. Fourier,) et D. Arnold (Penn. State Univ) pour des discussions stimulantes sur les modèles asymptotiques.

Centre de Recherches mathématiques et Département de Mathématiques et de Statistique, Université de Montréal, CP 6128, Succ. Centre-ville, MonTRÉAL (QuÉBEC), CANADA H3C 3J7

E-mail address: delfourocrm.UMontreal.ca 